\title{
Genome-wide and expression analysis of B-box gene family in pepper
}

\author{
Jing Ma ${ }^{\dagger}$, Jia-xi Dai ${ }^{\dagger}$, Xiao-wei Liu and Duo Lin ${ }^{*}$
}

\begin{abstract}
Background: BBX transcription factors are a kind of zinc finger transcription factors with one or two B-box domains, which partilant in plant growth, development and response to abiotic or biotic stress. The BBX family has been identified in Arabidopsis, rice, tomato and some other model plant genomes.

Results: Here, 24 CaBBX genes were identified in pepper (Capsicum annuum L.), and the phylogenic analysis, structures, chromosomal location, gene expression patterns and subcellular localizations were also carried out to understand the evolution and function of CaBBX genes. All these CaBBXs were divided into five classes, and 20 of them distributed in 11 of 12 pepper chromosomes unevenly. Most duplication events occurred in subgroup I. Quantitative RT-PCR indicated that several CaBBX genes were induced by abiotic stress and hormones, some had tissue-specific expression profiles or differentially expressed at developmental stages. Most of CaBBX members were predicated to be nucleus-localized in consistent with the transient expression assay by onion inner epidermis of the three tested CaBBX members (CaBBX5, 6 and 20).

Conclusion: Several CaBBX genes were induced by abiotic stress and exogenous phytohormones, some expressed tissue-specific and variously at different developmental stage. The detected CaBBXs act as nucleus-localized transcription factors. Our data might be a foundation in the identification of CaBBX genes, and a further understanding of their biological function in future studies.
\end{abstract}

Keywords: BBX, Pepper, Phylogenetic relationships, Subcellular localizations, Gene expression patterns

\section{Introduction}

In plant, transcription factors (TFs) are a kind of proteins that play an important part in physiological and biochemical processes by regulating the downstream gene transcription. There are usually four main domains for $\mathrm{TF}$ structure construction, all of which required for the functional process: DNA binding site, transcription activation domain, oligomerization site, and nuclear localization signal [1]. Among them, the study of B-box (BBX) zinc finger family is a growing area in recent years.

\footnotetext{
*Correspondence: linduo73@163.com

†Jing Ma and Jia-xi Dai contributed equally to this work.

Engineering Laboratory of Genetic Improvement of Horticultural Crops

of Shandong Province, Key laboratory of horticultural plant genetic

improvement and breeding of Qingdao, College of Horticulture, Qingdao

Agricultural University, 700 Changcheng Road, Qingdao 266109, China
}

The $\mathrm{BBX}$ transcription factors in plants usually carry one or two B-box domains $\left(\mathrm{CX}_{2} \mathrm{CX}_{8} \mathrm{CX}_{7} \mathrm{CX}_{2} \mathrm{CX}_{4} \mathrm{HX}_{8} \mathrm{H}\right)$ in the $\mathrm{N}$-terminal region, in which the conserved Cysteine $(\mathrm{C})$ and Histidine $(\mathrm{H})$ residues are predicted to be involved in protein-protein interactions [2]. The conserved B-box domains consisting of 40 amino acids was widely found in more than 1500 proteins of multicellular species and some unicellular eukaryotes [3]. Also, some plant BBX proteins are characterized containing an additional highly conserved CCT (CONSTANS, CO-like, and TIMING of CAB1) domain in the C-terminus [4, 5], which play an essential role in transcriptional regulation and nuclear transport $[6,7]$. There are 32 BBXs found in Arabidopsis, named AtBBX1 32 [2]. These AtBBX proteins were divided into five structure groups (Groups $\mathrm{I} \sim \mathrm{V}$ ) depending on the number and sequence features of 
the B-box domain or the presence of a CCT domain $[2,8$, 9]. Both Group I (AtBBX1 6) and II (AtBBX7 13) proteins possess two B-box domains and one CCT domain, with some differences at consensus sequences of second B-box domain between Group I and II [8]. AtBBX14 to AtBBX17 belonging to Group III had a single B-box domain in association with a CCT domain; the AtBBX members of structure group IV (AtBBX18 25) contain two B-box domains without CCT domain; while Group $\mathrm{V}$ proteins (AtBBX26 to AtBBX32) only had a single B-box domain [2]. Additionally, the BBX TFs were identified in several other model plants in recent years, such as 30 members in rice, 29 in tomato, 64 in apple, 39 in pear, and so on [10-14]. And the BBX TFs identified in all these model plants were classified into five groups in the same cases with Arabidopsis BBX members.

In plants, B-box (BBX) proteins are well-known to be involved in plant development, especially light, circadian signaling and flowering. $C O / A t B B X 1$ was the first discovered BBX protein, a core component which can promote flowering under long-day condition $[15,16]$. Other two BBX proteins, $B B X 2$ and $B B X 3$ were investigated to be less influenced on flowering time, but overexpression of $B B X 2$ gene showed a decreasing duration of two specific circadian rhythms in Arabidopsis [17]. BBX21 (also known as SALT TOLERANCE HOMOLOG 2) were identified as a regulator of several $A B A I N S E N$ SITIVE $(A B I)$ genes and directly activates ELONGATED HYPOCOTYL 5 (HY5) in ABA control of seed germination, which is targeted by COP1 for $26 \mathrm{~S}$ proteasomemediated degradation in Arabidopsis [18-20]. AtBBX28 was found that interact with both HY5 and COP1 at its $\mathrm{C}$-terminal portion resulting in negatively regulates photomorphogenic development [21]. Besides, HY5 negatively regulated $\mathrm{BBX} 30$ and $\mathrm{BBX} 31$ by directly binding to the G-box cis-element present in their promoters, negatively regulate photomorphogenesis in Arabidopsis [22]. BBX4 is a key component involved in the phyB (Phytochrome B)-PIF3 (PHYTOCHROME INTERACTING FACTOR 3) regulatory module. phyB directly interacts with BBX4 and positively controls the level of BBX4 protein in red light. And BBX4 repressed the transcriptional activation activity of PIF3 by directly interacting with PIF3, thereby promoting photomorphogenesis [23]. And BBX11-BBX21-HY5 can positively regulate photomorphogenesis in the response to light during normal development [24]. Besides, several BBX are identified to be involved in flowering by positively and negatively regulating the $C O$ (CONSTANS) and $F T$ (Flowering Locus T) genes expression [25-29]. In rice, $\mathrm{OsCO} 3$ possessing a single B-box and CCT domain functions as a negative FT-like genes regulator which delays flowering time under SD (short day) conditions [30]. OsCLO4 showed a represses flowering under SD and LD (long day) conditions [31]. And $H d 1$ (OsBBX18) containing two B-box motifs and one additional CCT domain, promote flowering under SD conditions and inhibit under LD conditions $[4,32]$. Nevertheless, several BBXs in other plants, such as barely (Hordeum vulgare), beetroot (Beta vulgaris), chrysanthemum (Chrysanthemum morifolium), and grape (Vitis vinifera), also play an important role in regulation of flowering [4, 30, 33].

In addition, $B B X$ family genes have shown their roles in mitigating abiotic stresses. The salt tolerance protein (STO, AtBBX24) was first identified to trigger the salt tolerance activities in yeast cells [34], which can enhance Arabidopsis root growth under salt stress treatment [35]. STO negatively regulated a wide range of stressrelated genes [36], which can also interact with CLONE EIGHTY-ONE/RADICAL INDUCED CELL DEATH1 (CEO/RCD1) [37, 38]. AtBBX18 was detected to be a negative regulator both in photomorphogenesis and heat tolerance in Arabidopsis [39]. In rice and tomato, most promoters of the $O s B B X$ and $S l B B X$ genes contain at least one stress-responsive cis-element (ARE, Wbox, GC-motif, Box-W1, HSE, and MBS). Through the expression analysis under biotic or abiotic stress, the expression levels of most of BBX family members in the rice and tomato were significantly changed under most treatments, indicating that these genes were induced by biotic or abiotic stress [10, 11]. In Chrysanthemum, CmBBX24 not only play a part in delaying flowering time, but also enhance cold and drought tolerance in plant [33]. Besides, overexpression of $V v B B X 32$ can increase cold tolerance in transgenic Arabidopsis plants [40]. Recently, an apple B-box protein BBX37 was identified that regulates jasmonic acid mediated cold tolerance through the JAZ-BBX37-ICE1-CBF pathway [41]. Although the studies of $B B X$ transcription factors are increasing rapidly in previous years, there are few studies about $B B X$ genes in pepper [42]. And the whole genome sequencing of Capsicum annuum L. makes it possible for analyzing deeply in the $B B X$ gene family of pepper $[43,44]$.

Pepper (Capsicum annuum L.) is a dominant vegetable species belonging to Solanaceae family cultivated all over the world. And in recent one or two years, it has overtaken tomato, with the cultivated area in first place in the world. However, the vegetative and reproductive growth of pepper was negatively affected by biotic and abiotic stresses such as salt, cold, heat, drought, diseases and insect pests. And BBX are thought to play important roles in plant abiotic and biotic stress responses, thus the study of CaBBX TFs in these molecular mechanisms is necessary to determine the biological processes involved in the multiple regulatory of abiotic tolerance. In this study, $24 \mathrm{CaBBX}$ members were identified in pepper. And 
we also performed the gene structure, phylogenetic relationships, chromosome localization, subcellular localizations and their expression patterns under various abiotic stresses and hormones treatments in pepper.

\section{Materials and methods}

\section{Identification of BBX family genes in pepper}

We firstly obtained the conserved B-box domain (PF00643) based on a Hidden Markov Model (HMM) from the Pfam database (Pfam 32.0, http://pfam.xfam. org/). Then the HMM profile of the B-box domain was utilized to do BLASTP search by using HMMER 3.2 in pepper genome databases with an expected value (e-value) cut-off of $0.01[43,44]$. Afterwards, the putative CaBBX proteins obtained were confirmed for the presence of the B-box domain by the SMART (http://smart. embl-heidelberg.de/) and Pfam (http://pfam.xfam.org/) searches and InterProscan (http://www.ebi.ac.uk/inter pro/search/sequence-search) programs. In addition, the isoelectric point $(\mathrm{pI})$ and molecular weight $(\mathrm{kDa})$ of the obtained CaBBX proteins were determined by using the ExPASy proteomics server (https://web.expasy.org/) [45].

\section{Phylogenetic analysis and sequence alignment}

The BBX sequences of tomato were obtained from the NCBI database (http://www.ncbi.nlm.nih.gov/). Multiple sequence alignments of $\mathrm{CaBBX}$ proteins were carried out with the ClustalX program (Version 2.1) [46]. The p-distance-based phylogenetic tree was constructed with the neighbor-joining algorithm in MEGA (version 7.0) with a bootstrap value of 1000 [47].

\section{Domains, motif structure and gene structure analysis}

Domains were identified with Conserved Domain Database (CDD) in NCBI (https://www.ncbi.nlm.nih.gov/ cdd). MEME Suite was used to determine all motifs in the CaBBX protein sequences [48]. Analysis was performed using the following parameters: number of repetitions, if any; optimum width of the motif, 6-50; and maximum number of motifs, 8 . And the intron and exon were determined by CDS and genomic information in pepper Genome Database (http://pepperhub.hzau.edu. $\mathrm{cn} /$ ). All these structures were visualized by TBtools [49].

\section{Chromosomal location and duplication analysis of $\mathrm{CaBBXs}$}

The identified $C a B B X$ gene annotations and their chromosomal locations were retrieved from genome annotations downloaded from the Pepper Genome Database (http://pepperhub.hzau.edu.cn/) according to the gene ID. The exact location of genes on chromosomes was drawn by using TBtools. Duplication analysis was also constructed by using TBtools [49].
Plant materials, growth condition, hormone and stress treatments to plants

Pepper seeds ('Qingnong dried No.2') were obtained from the State Key Laboratory of Crop Genetics and Germplasm Enhancement in Qingdao Agricultural University. This cultivar was selected by researchers at Qingdao Agricultural University (Qingdao, China), the Qingdao Seed Station, and Dezhou Academy of Agricultural Sciences. Additionally, it was approved by the Shandong Variety Examination and Approval Committee in 2015(deposition number: 2015-057-1). At first, the seeds were germinated in light incubator at $28^{\circ} \mathrm{C}$. Three days later, the germinated seeds were transplanted into pot in a growth chamber with a photoperiod of $14 \mathrm{~h}$ of light and $10 \mathrm{~h}$ of darkness at $28^{\circ} \mathrm{C} / 21^{\circ} \mathrm{C}$. Six-leaf seedlings were used to treat with $100 \mu \mathrm{M}$ Abscisic acid (ABA), $100 \mu \mathrm{M}$ Methyl jasmonate (MeJA), $100 \mu \mathrm{M}$ Salicylic acid (SA), $10 \%$ polyethylene glycol-6000 (PEG-6000), and $100 \mathrm{mM}$ $\mathrm{NaCl}$. High and low temperature were applied by placing seedlings in 38 and $4{ }^{\circ} \mathrm{C}$ growth chamber, respectively. The leaf tissues were harvested at $0,3,6$ and $12 \mathrm{~h}$ post various treatments. And all collected samples were immediately frozen in liquid nitrogen, and then stored at $-80^{\circ} \mathrm{C}$ for RNA isolation. All samples were collected in triplicate from each of the sampling points. Besides, the samples of root, stem, leaf, flower, fruit and seed were harvested to investigate the tissue-specific expression.

\section{Total RNA isolation and CDNA synthesis}

Total RNA was isolated from plant materials using a total RNA kit (Tiangen, Beijing, China) according to the manufacturer's instruction. A total of $1 \mu \mathrm{g}$ of RNA of each sample was used for first-strand cDNA synthesis using M-MLV reverse transcriptase according to the manufacturer's protocols (TaKaRa, Dalian, China). cDNA was diluted 20-fold for qRT-PCR analysis.

\section{Quantitative real-time PCR}

Primers were designed based on $C a B B X$ gene sequences for real-time PCR by using the real-time PCR design tool in Integrated DNA Technologies (IDT, https://sg.idtdna. com/scitools/Applications/RealTimePCR/) (All primers are listed in Table S1). Real-time PCR application was carried out in a LightCycler ${ }^{\circledR} 480$ Real-Time PCR Detection System (Roche, Hercules, Switzerland) with ChamQ SYBR Color qPCR Master Mix (Vazyme, Nanjing, China). The constitutive actin gene (Gen Bank accession No. AY572427) was used as an internal control and served as a standard gene for normalizing all mRNA expression levels [50]. A total of $20 \mu \mathrm{L}$ reaction system contained $10 \mu \mathrm{L}$ SYBR Color qPCR Master Mix, $1 \mu \mathrm{L}$ cDNA samples, $0.4 \mu \mathrm{L}$ of each primer $(10 \mu \mathrm{M})$ and $8.2 \mu \mathrm{L} \mathrm{ddH}_{2} \mathrm{O}$. 
The PCR thermal cycle conditions were as follows: denaturation at $95^{\circ} \mathrm{C}$ for $30 \mathrm{~s}, 40$ cycles of $95^{\circ} \mathrm{C}$ for $10 \mathrm{~s}$, and $58^{\circ} \mathrm{C}$ for $20 \mathrm{~s}$ and $72{ }^{\circ} \mathrm{C}$ for $20 \mathrm{~s}$. Fluorescence intensities were measured for $\mathrm{qRT}-\mathrm{PCR}$ at the end of each cycle. A melting curve $\left(61\right.$ cycles at $65^{\circ} \mathrm{C}$ for $10 \mathrm{~s}$ ) was performed directly to check for specific amplification. The relative gene expression was calculated by using the $2-\triangle \triangle \mathrm{Ct}$ method [51], the experiments were performed triplicate technological repeats. The SPSS statistics software (version 17.0) was used to analyze significant differences [52].

\section{Subcellular localization analysis}

The subcellular localization of CaBBX proteins was predicted by Plant-mPLoc in Cell-PLoc 2.0 (http://www. csbio.sjtu.edu.cn/bioinf/plant-multi/) [53]. And four CaBBX proteins were chosen to verify the predication results. The four selected $C a B B X$ genes were isolated from the cDNAs of pepper var. 'Qingnong dried No.2', and the amplified products were recombined into pMDC83 vector with green fluorescent protein (GFP), and then transferred to Agrobacterium tumefaciens Gv3101 strain for following infection. The onion inner epidermis was used for transforming with vectors with $C a B B X$ genes. Fluorescence images were captured and analyzed using a Zeiss laser scanning confocal microscope TCS SP5 (Leica, Brunswick, Germany) and the LSM image software.

\section{Results}

Identification and characteristics of $B B X$ genes in pepper We searched PepperHub (Pepper Information Hub, http://pepperhub.hzau.edu.cn/) and PGP (Pepper Genome Platform, http://passport.pepper.snu.ac.kr/?t= PGENOME) with the conserved B-box domain HMM profile (PF00643) to obtain global putative $B B X$ genes in pepper. Then the putative encoding protein sequences of these genes were further confirmed their B-box domain by using SMART, Pfam and InterProScan searches, six putative genes without B-box domain were removed. In total, we eventually identified $24 \mathrm{BBX}$ genes in pepper, which were named $C a B B X 1$ to $C a B B X 24$. Afterwards, the detailed information gene name, gene annotation ID, genomic position, gene length, theoretical isoelectric point, and molecular weight of their encoding protein were listed in Table 1.

These $B B X$ genes showed diverse in length leads to the various length, theoretical isoelectric point, and molecular weight of their encoding protein. These $B B X$ genes with sequence of 582 to $1476 \mathrm{bp}$ encoded ranging from 193 (least, $C a B B X 7$ ) to 491 (most, $C a B B X 22$ ) amino acid residues. And the isoelectric points of $24 \mathrm{BBX}$ proteins were ranging from 4.60 (lowest, $C a B B X 23$ ) to
9.17 (highest, $C a B B X 24$ ), with the molecular weights of $21.20 \sim 54.98 \mathrm{kDa}$ (Table 1).

\section{Phylogenetic analysis of the CaBBX family}

To identify the phylogenetic relationship and division of $\mathrm{CaBBX}$ proteins, we constructed the phylogenetic tree of BBX family proteins in pepper (Fig. 1). The phylogenetic analysis of the CaBBXs with AtBBXs, PtBBXs, OsBBXs and SIBBXs was also carried out to confirm the subfamily clustering of $\mathrm{CaBBX}$ (Fig. S1). The division of $24 \mathrm{CaB}-$ $\mathrm{BXs}$ were not even on the phylogenetic tree (Fig. 1A). All the 24 CaBBXs were divided into five subfamilies with the similarity of the amino acid sequences based on previous studies in tomato [1]. In addition, the phylogenetic relationship of first B-box domain was constructed, as well as two B-box and one CCT domain (Fig. 1B and C). In total, there were eight $\mathrm{CaBBX}$ s classified into subclass I, whose contain two B-box domains, making up the largest subclass. The subclass II and III both contained six members, and only two members (CaBBX12 and 13) clustered together in subclass IV, and CaBBX23 and 24 aligned together in subclass V. Members from subclass II owed two B-box domains and one CCT domain, while only one BBX proteins ( $\mathrm{CaBBX} 13)$ possessed one B-box and one CCT domain belonging to subclass IV. Other CaBBX proteins only contained one or two B-box domains without CCT domain. Moreover, based on the phylogeny of BBXs in Arabidopsis, rice, tomato and Populus tomentosa, most of the BBXs with two B-box domains and one CCT domain were classified into subgroup II, and most of whom with two B-box domains and none CCT domain were classified into subgroup I. While, BBXs contain one single B-box domain were most together classified into subgroup $\mathrm{V}$.

\section{Domains, motif structure and gene structure analysis}

To determine the domains, motif structure and gene structure of $\mathrm{CaBBXs}$, the conserved domain information were confirmed by CDD in NCBI, and motif and CDS were also plotted to identify structure analysis of CaBBXs (Fig. 2).

Eight motifs were identified in these CaBBXs, members from same subclass shared similar motifs according to the phylogenetic relationship (Fig. 2A). For example, all the members from subclass I contained motif 1,3 and 7 , only CaBBX20 also owed another motif 6 . While, except CaBBX4 only owed two motif 1 and one motif 2 , CaBBXs from subclass II possessed maximum motifs, containing motif 1, 2, 3, 5 and 7; moreover, three of them (CaBBX1, 2 and 3) contained all the 8 identified motifs. Other members carried one or two motif 1 , several of them contained motif 2; in addition, CaBBX11 and 13 also had a motif 4 and 8 located in middle of their amino 
Table 1 Information of the BBX gene family in pepper

\begin{tabular}{|c|c|c|c|c|c|c|c|c|}
\hline Gene & Annotated CDS & Genomic position & Chr & CDS & AA & pls & MV & $\begin{array}{l}\text { Subcellular } \\
\text { localization }\end{array}$ \\
\hline CaBBX1 & Capana02g003201 & $157,124,787-157,126,407$ & 2 & 1224 & 407 & 5.27 & 43.89 & Nucleus \\
\hline CaBBX2 & Capana02g003200 & $157,118,313-157,120,062$ & 2 & 1197 & 398 & 5.32 & 45.39 & Nucleus \\
\hline CaBBX3 & Capana02g003199 & $157,107,846-157,110,095$ & 2 & 1215 & 404 & 5.41 & 44.56 & Nucleus \\
\hline CaBBX4 & Capana01g004030 & $278,243,944-278,245,872$ & 1 & 1032 & 343 & 5.45 & 37.93 & Nucleus \\
\hline CaBBX5 & Capana12g000414 & $8,179,392-8,181,909$ & 12 & 1071 & 356 & 5.29 & 39.43 & Nucleus \\
\hline CaBBX6 & Capana07g000030 & $1,563,552-1,565,426$ & 7 & 1155 & 384 & 6.56 & 42.54 & Nucleus \\
\hline CaBBX7 & Capana00g004028 & $606,409,675-606,410,256$ & - & 582 & 193 & 5.32 & 21.20 & Nucleus \\
\hline CaBBX8 & Capana07g001114 & $154,056,816-154,057,654$ & 7 & 693 & 230 & 5.03 & 24.83 & Nucleus \\
\hline CaBBX9 & Capana00g004489 & $649,683,777-649,686,080$ & - & 1281 & 426 & 5.07 & 46.87 & Nucleus \\
\hline CaBBX10 & Capana03g003558 & $228,734,754-228,738,294$ & 3 & 1164 & 387 & 5.45 & 43.34 & Nucleus \\
\hline CaBBX11 & Capana00g001486 & $399,610,115-399,614,902$ & - & 1410 & 469 & 6.75 & 51.67 & Nucleus \\
\hline $\mathrm{CaBBX} 12$ & Capana11g002294 & $218,148,220-218,150,401$ & 11 & 951 & 316 & 4.85 & 35.20 & Nucleus \\
\hline CaBBX13 & Capana03g000377 & $5,273,465-5,275,489$ & 3 & 1149 & 382 & 5.53 & 43.46 & Nucleus \\
\hline CaBBX14 & Capana02g002620 & $148,180,402-148,183,481$ & 2 & 624 & 207 & 8.47 & 22.97 & Nucleus \\
\hline CaBBX15 & Capana08g002625 & $149,926,925-149,929,248$ & 8 & 639 & 212 & 6.17 & 23.52 & Nucleus \\
\hline CaBBX16 & Capana12g000659 & $18,213,299-18,215,385$ & 12 & 960 & 319 & 6.50 & 35.22 & Nucleus \\
\hline CaBBX17 & Capana04g000266 & $4,091,269-4,092,722$ & 4 & 918 & 305 & 6.24 & 33.86 & Nucleus \\
\hline CaBBX18 & Capana07g002062 & $212,563,126-212,570,473$ & 7 & 894 & 297 & 4.98 & 32.01 & Nucleus \\
\hline CaBBX19 & Capana09g000394 & $12,877,150-12,887,068$ & 9 & 900 & 299 & 4.97 & 32.17 & Nucleus \\
\hline CaBBX20 & Capana06g000735 & $11,697,317-11,699,646$ & 6 & 702 & 233 & 5.00 & 25.98 & Nucleus \\
\hline CaBBX21 & Capana08g002611 & $149,745,414-149,746,937$ & 8 & 804 & 267 & 5.62 & 29.83 & Nucleus \\
\hline $\mathrm{CaBBX} 22$ & Capana05g001195 & $84,622,805-84,628,926$ & 5 & 1476 & 491 & 6.00 & 54.98 & Nucleus \\
\hline $\mathrm{CaBBX} 23$ & Capana07g001588 & $191,773,542-191,774,577$ & 7 & 744 & 247 & 4.60 & 27.40 & Nucleus \\
\hline CaBBX24 & Capana00g004911 & $672,862,631-672,863,374$ & - & 744 & 247 & 9.17 & 27.71 & Nucleus \\
\hline
\end{tabular}

Note:Annotated CDS annotated coding DNA sequences, Genomic position, Chr chromosome, CDS coding DNA sequences, AA amino acid residues, pl theoretical isoelectric point, MV, Subcellular localization. The subcellular location results of pepper BBX genes were predicted by Plant-mPLoc in Cell-PLoc 2.0

acid sequence, respectively. The detail sequence information of these eight motifs were shown in Fig. S2.

Furthermore, the gene structures of $C a B B X s$ were constructed with TBtools by gff file from pepper genome 2.0 [49]. Among 24 CaBBXs, only CaBBX24 had no intron, others had one to five exons. To make clear the domains arrangement, we also plotted domains on the CDS directly. Nine BBX proteins were identified containing two B-box and a CCT domain, five of them share two same B-box, and four possessed two different B-box domains. Only one BBX proteins (CaBBX13) possessed one B-box and one CCT domain, while three and eleven CaBBX proteins contained one and two B-box without CCT domain, respectively (Fig. 2B). These results were consistent with the phylogenetic divergence analysis. Except for subclass I members, the B-box domains of members from other subclasses were located in the beginning of first exon. The B-box domains of subclass I members were on the first three exon. CCT domain were situated in the terminal of last two exon. Moreover, the two B-box (B-box1 and B-box2 domains) share similar conserved sequences and Zinc finger domain.

\section{Chromosomal localization and duplication of $B B X$ genes in pepper}

We have plotted the $C a B B X$ genes to the chromosomes of pepper genome to confirm their genomic distribution (Fig. 3). Except for four $C a B B X$ genes ( $C a B B X 7, C a B B X 9$, $C a B B X 11$ and $C a B B X 24), 20 C a B B X$ genes were distributed unevenly on 11 of 12 pepper chromosomes, no gene was on chromosome 10. Both chromosome 02 and 07 possessed four $C a B B X$ genes, making up the maximum number of genes among all these 12 chromosomes. In addition, only one $C a B B X$ gene was located on chromosome $01,04,05,06,09$ and 11, respectively; and two on chromosome 03,08 and 12 , respectively.

Potential duplication within pepper were marked on the 12 chromosomes by using TBtools [49]. Expect $C a B B X 7$ (duplicated with $C a B B X 8$ ) was not located on pepper chromosomes, the other three duplications only occurred on the 3 of 12 chromosomes, and these duplicated genes (CaBBX14, CaBBX15, CaBBX17 and CaBBX21) were all belonged to subgroup I (Fig. 3). And all the duplication events occurred between two different chromosomes, not within the same chromosome. 


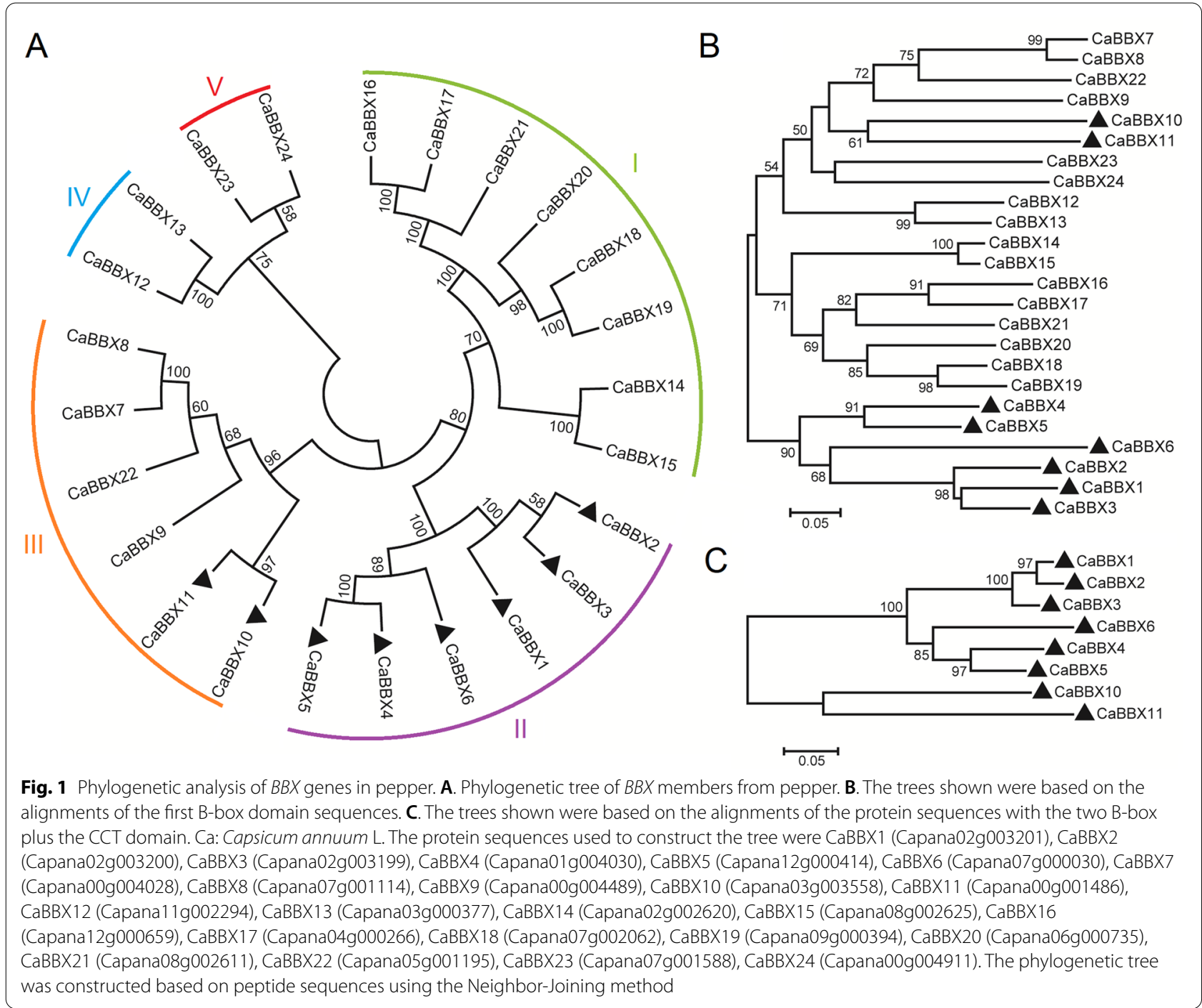

In addition, we constructed a collinearity relationship analysis to identify the duplication events of BBX genes between pepper and the model Solanaceae plant tomato (Fig. 4). Twenty-six pairs of BBX genes were identified duplicate between pepper genome and tomato genome. All the subgroups of $B B X$ genes involved in duplication. Among them, 13 pairs of subgroup I members play part in the replication events, account for half of the total duplication events. And we found 4 pairs of subgroup II and III members, 3 pairs of subgroup IV members and 2 pairs of subgroup $\mathrm{V}$ members were homologous in the pepper and tomato genome (Fig. 4).

\section{Organ development expression analysis of $B B X$ genes in pepper}

To investigate the tissue-specific and developmental expression pattern of all the $C a B B X$ genes, we performed the heatmap by using TBtools based on the transcript data from Pepper Information Hub (http://pepperhub. hzau.edu.cn/) [49]. Several CaBBX genes showed organspecific expression pattern, such as CaBBX19, expressed specifically in seed, respectively, and expressed arise as the tissues' development (Fig. 5). This result indicated that CaBBX19 may play an important role in seed morphogenesis development, respectively. CaBBX7, $C a B B X 12, C a B B X 13$ and $C a B B X 22$ mainly expressed in leaf, may showed their regulatory function in pepper leaf (Fig. 5). Additionally, CaBBX5 and CaBBX6 showed high expression levels in leaf and flower, $\mathrm{CaBBX} 3$ and CaBBX14 specifically expressed in the early developmental stage of flower, CaBBX4 and CaBBX20 expressed in almost all the detected tissues, and expressed most highly in fruit development, especially in the pericarp, however, expect in the seed (Fig. 4). This result may indicate that 

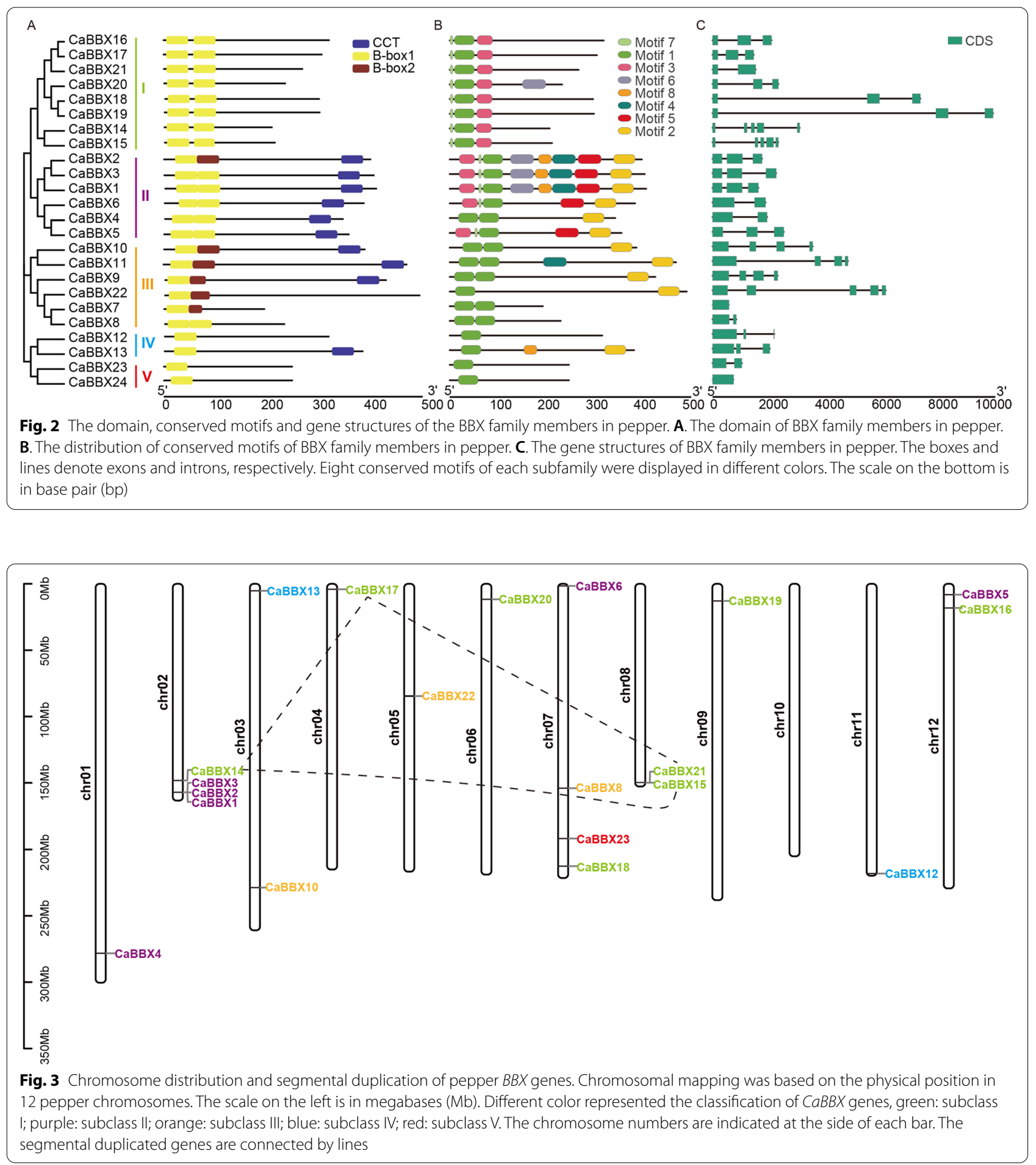

$C a B B X 4$ and $C a B B X 20$ involved in the pericarp development (such as pigmentation, enlargement, and so on).

Furthermore, we also investigated the expression levels of 24 CaBBX genes by qRT-PCR. In particularly,
$C a B B X 19$ showed the highest expression level in seed, and $C a B B X 24$ was expressed more highly in flower than that in the other tissues, indicating that $C a B B X 19$ and $C a B B X 24$ play essential roles in controlling pepper 

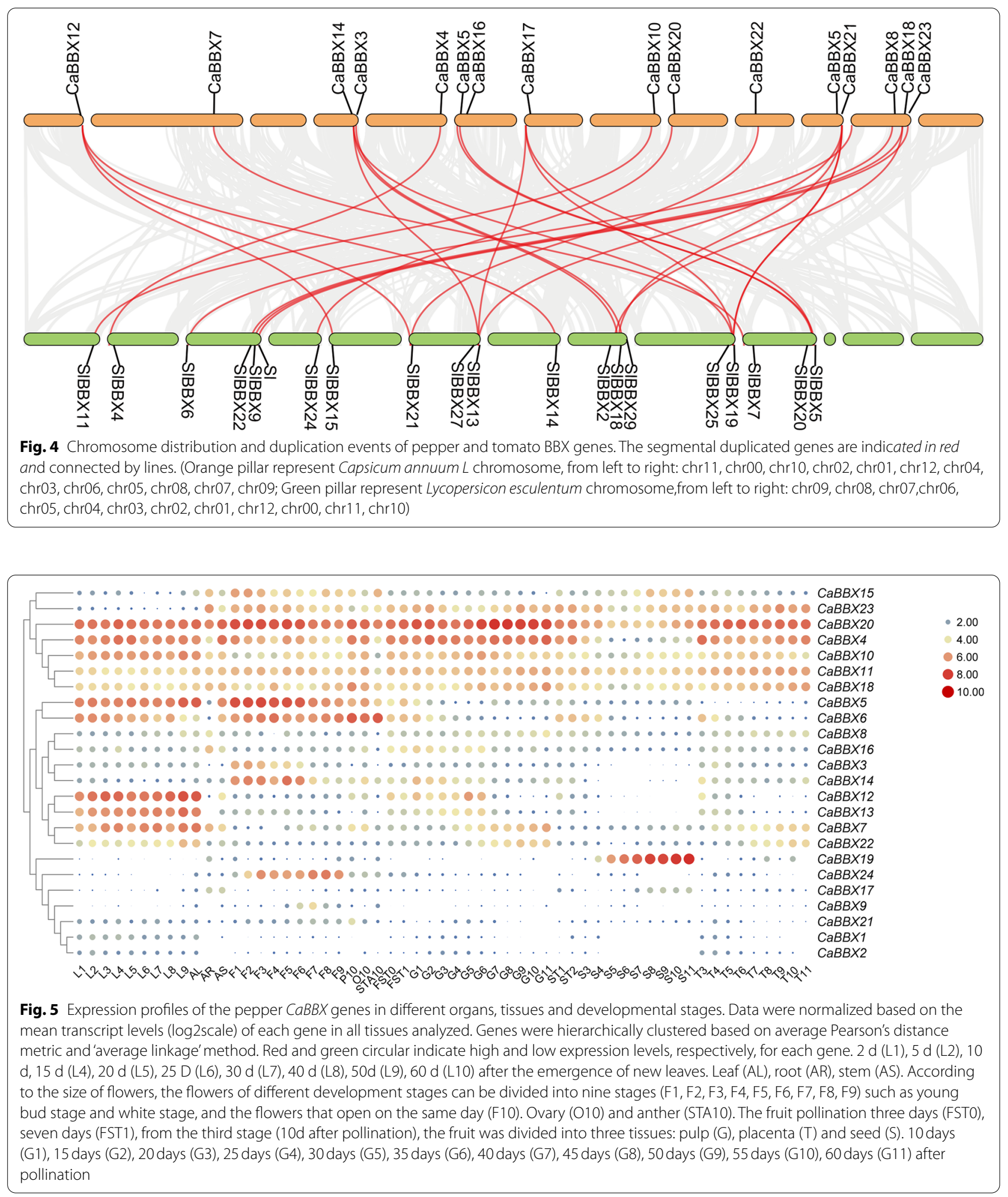

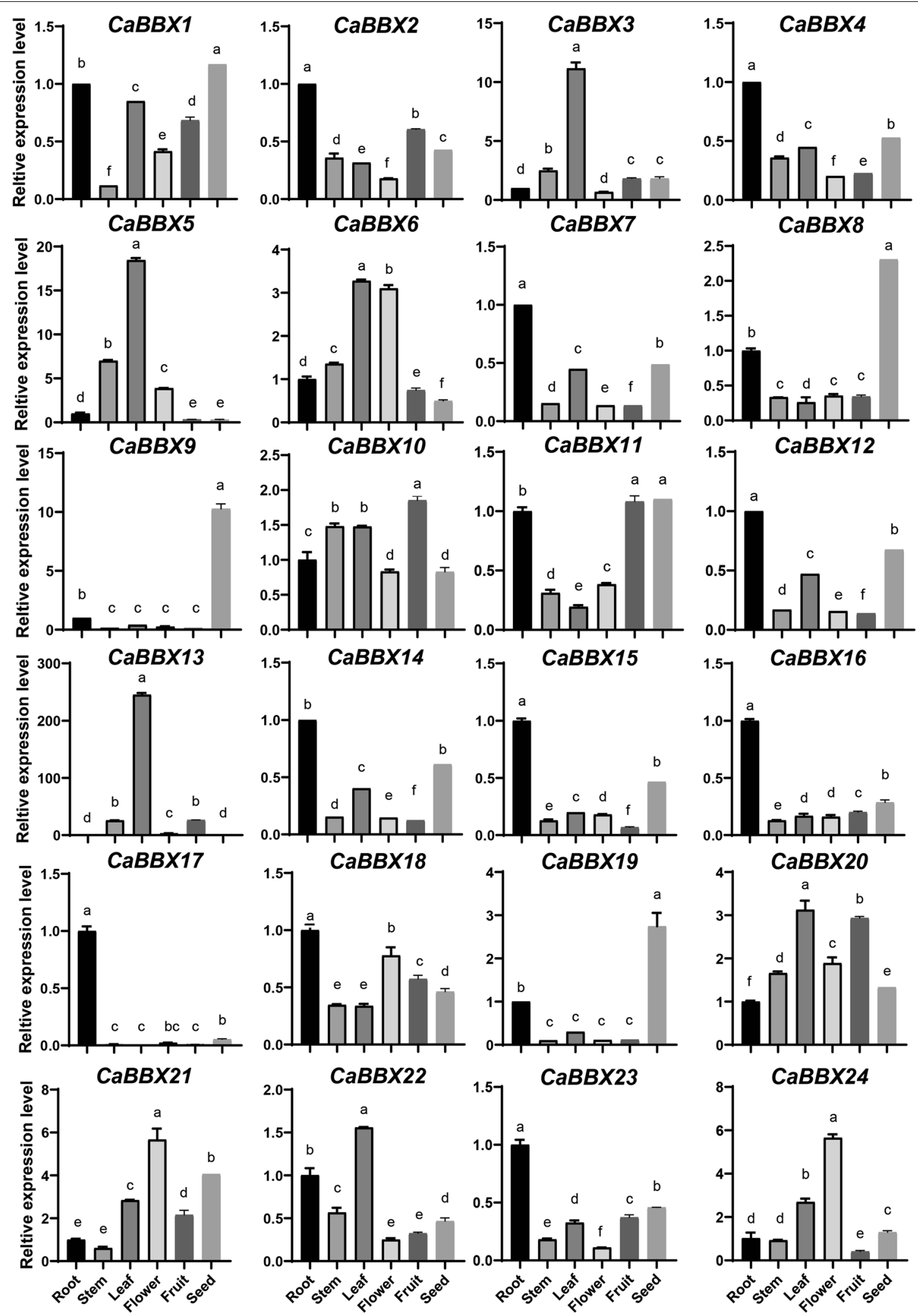

Fig. 6 The expression of CaBBXS in different pepper tissues. Different tissues were arranged as 'root, stem, leaf, flower, fruit and seed'. Three independent biological experiments were performed $(P<0.05)$ 
seed development and flowering (Fig. 6). CaBBX13 and 22 showed highly expression in leaf, while CaBBX6 expressed highly in both leaf and flower. These results were consisting with that of RNA-seq data transcript levels.

\section{Expression analysis of $B B X$ genes under abiotic stress in pepper}

The expression levels of $C a B B X$ genes under cold, heat, salt and drought stress were investigated by qRT-PCR analysis, to analyze $C a B B X$ genes in response to abiotic stress (Fig. 7). We selected six $C a B B X$ genes (CaBBX3, CaBBX4, CaBBX5, CaBBX6, CaBBX13 and CaBBX20) potentially responding to abiotic stress base on transcriptome data of different stress treatment from the Pepper Genome Database (Fig. S3, http://pepperhub.hzau. edu.cn/). The expression levels were detected under high temperature, low temperature, $\mathrm{NaCl}$ and PEG6000 treatment at $3 \mathrm{~h}, 6 \mathrm{~h}, 12 \mathrm{~h}$. In low temperature treatment, four of these six $C a B B X$ genes showed up-regulated expression, except for $C a B B X 13$ and $C a B B X 20$, they both showed a high expression level under $3 \mathrm{~h}$ cold treatment, but rapidly down-regulated afterwards $6 \mathrm{~h}$ (Fig. 7A). Under heat stress condition, the expression of $C a B B X 4, C a B B X 5, C a B B X 6$ and $C a B B X 13$ got a peak at 3 or $6 \mathrm{~h}$ treatment, and then decreased at $12 \mathrm{~h}$ treatment, while $C a B B X 3$ and $C a B B X 20$ exhibited the opposite expression pattern (Fig. 7B). There were three $C a B B X$ genes ( $C a B B X 4, C a B B X 5$ and $C a B B X 6$ ) expressed up-regulated under drought stress, and the expression levels of other three genes were decreased (Fig. 7C). Only the expression of $C a B B X 3$ were repressed under salt condition, and showed an early down-regulation at 3 and $6 \mathrm{~h}$, but rapidly increased its expression even more so than the control at $12 \mathrm{~h}$, while $C a B B X 4, C a B B X 5$, $C a B B X 6$ and $C a B B X 20$ were expressed more highly than control. Additionally, $C a B B X 13$ showed an up-regulation at 3 and $6 \mathrm{~h}$, but rapidly decreased with a more lower expression level than control at $12 \mathrm{~h}$ treatment (Fig. 7D). These results indicated that the six $C a B B X$ genes may involve in responding to abiotic stress.

\section{Expression analysis of $\mathrm{CaBBX}$ genes in response to exogenous hormones}

In addition, the expression pattern of $C a B B X$ genes under ABA, MeJA and SA treatment were also measured because of their important part in plant growth, development and in response to biotic and abiotic stress.
The expression profiles of six $C a B B X$ genes potentially involved in response to abiotic stress were also investigated under $\mathrm{ABA}, \mathrm{MeJA}$ and SA treatment at $3 \mathrm{~h}, 6 \mathrm{~h}$, $12 \mathrm{~h}$ (Fig. 8). Three of these detected genes (CaBBX4, $C a B B X 13$ and $C a B B X 20$ ) were induced to express more highly by $A B A$ treatment than control at different timepoint treatment. $C a B B X 4$ was up-regulated during the entire treatment. However, the expression level of $C a B B X 13$ was increased significantly at 3 and $6 \mathrm{~h}$ treatment, but was repressed at $12 \mathrm{~h}$ treatment. And the expression of $C a B B X 3$ was repressed by ABA significantly at all the three time-point $(3,6$ and $12 \mathrm{~h})$ treatment. $C a B B X 5$ showed no obviously significant expression levels, and the expression of $C a B B X 6$ at 3 and $12 \mathrm{~h}$ treatment was lower than that of control (Fig. 8A). The expression of $C a B B X 3, C a B B X 4$ and $C a B B X 20$ were repressed dramatically after MeJA application, while the expression of other three genes ( $C a B B X 5, C a B B X 6$ and $C a B B X 13)$ were up-regulated at early ( $3 \mathrm{~h}$ and/or $6 \mathrm{~h}$ ) treatment stage, but $C a B B X 6$ and $C a B B X 13$ were expressed decreased rapidly at $12 \mathrm{~h}$ treatment (Fig. 8B). Except for $C a B B X 3$ and $C a B B X 13$, all the other four selected $C a B B X$ genes were induced to expressed at a higher level by exogenous SA, and reached peak at $6 \mathrm{~h}$ treatment. $C a B B X 13$ was also up-regulated under early treatment stage, but down-regulated under later treatment stage (Fig. 8C).

\section{Subcellular localization of CaBBXs}

The subcellular localization of proteins was analyzed to further understand the function. We first predicted the subcellular localization by Plant-mPLoc in Cell-PLoc 2.0 (Table 1). All of the 24 CaBBXs were identified to be located in nucleus with the highest possibility. We selected three CaBBXs (CaBBX5, 6, and 20) which were strongly induced or repressed by abiotic stress, hormones or showed organ-specific and organ developmental expression patterns for a transient expression assay using GFP-fused BBX proteins with onion epidermis. All the three CaBBXs were found to be in the nucleus (Fig. 9). The result of CaBBX20, CaBBX5 and 6 was consistent with the most preferentially predication.

\section{Discussion \\ Genome evolution of the B-box transcription factors in pepper}

BBX transcription factors were widely identified in many higher plants, such as Arabidopsis [2], rice [11],

(See figure on next page.)

Fig. 7 The expression of CaBBXs under different abiotic stresses. A. qRT-PCR transcript analysis of 6 selected CaBBX genes under cold stress. B. qRT-PCR transcript analysis of 6 selected $C A B B X$ genes under heat stress. $\mathbf{C}$. qRT-PCR transcript analysis of 6 selected $C a B B X$ genes under drought stress. D. qRT-PCR transcript analysis of 6 selected $C A B B X$ genes under salt stress. Three independent biological experiments were performed $(P<0.05)$ 

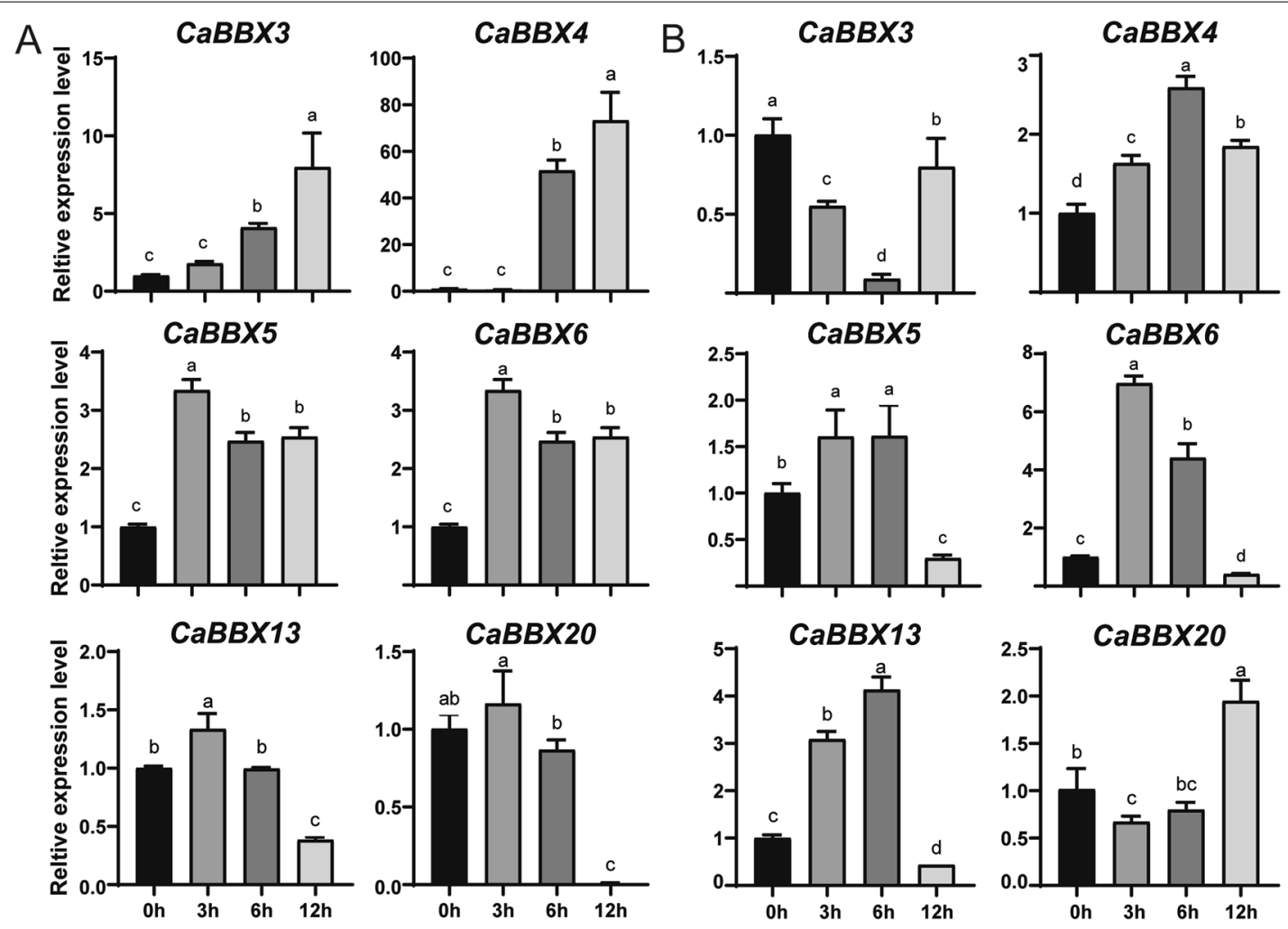

C
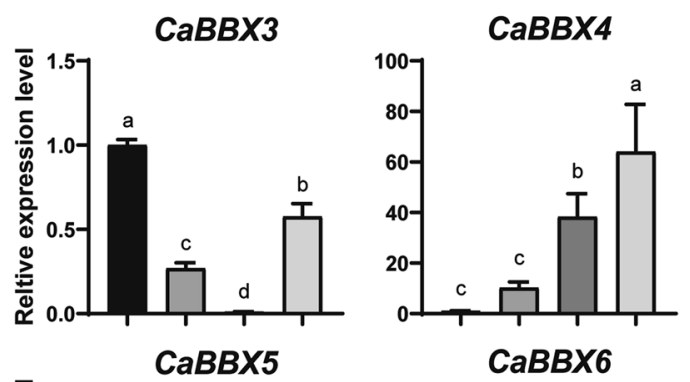

D CaBBX3

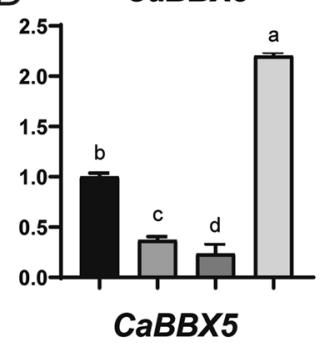

CaBBX4
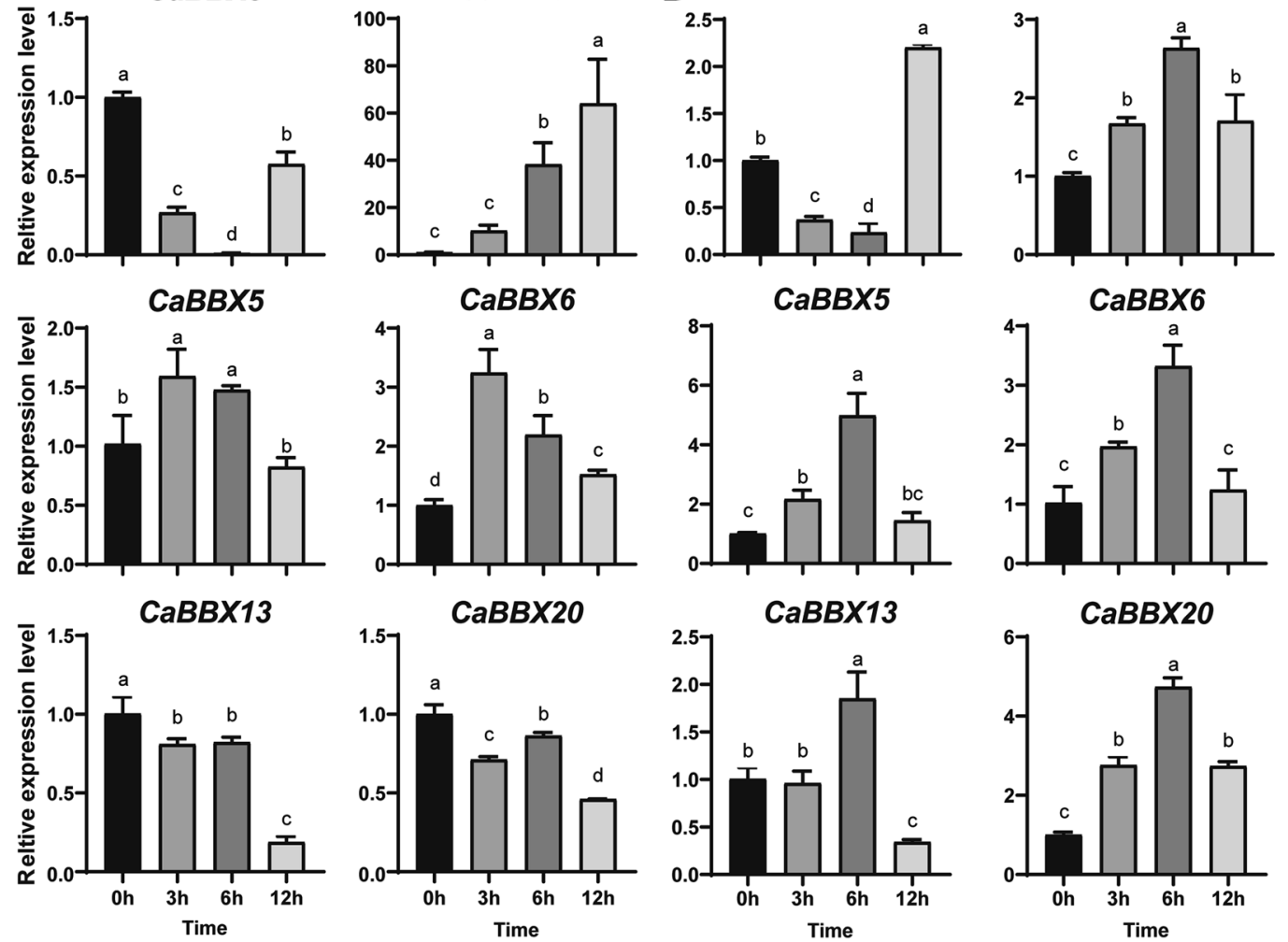

Fig. 7 (See legend on previous page.) 

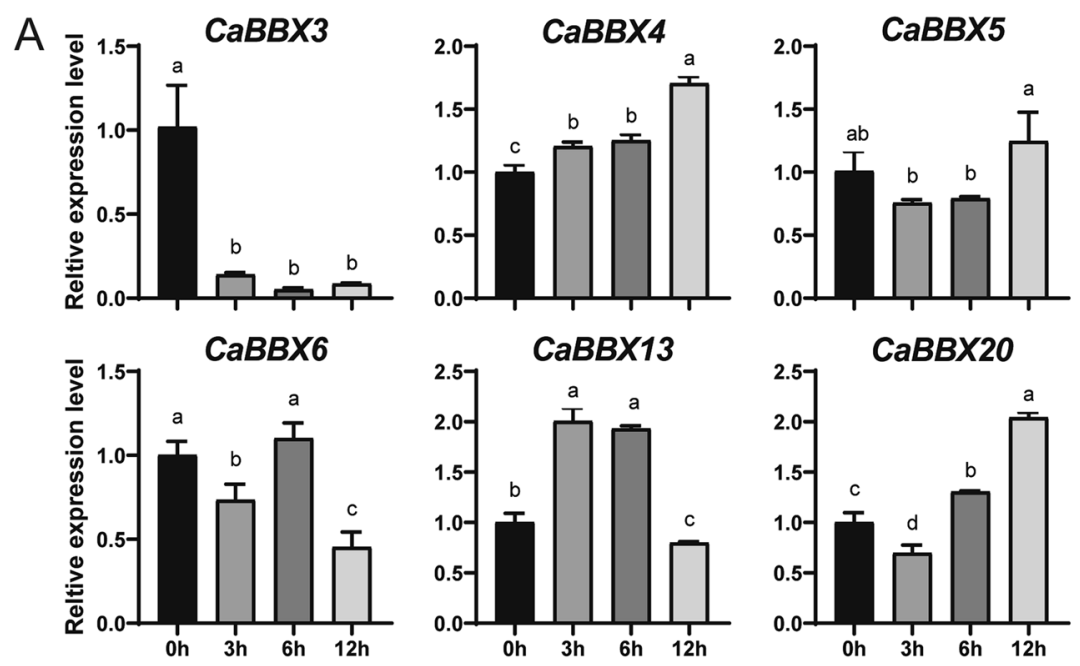

B
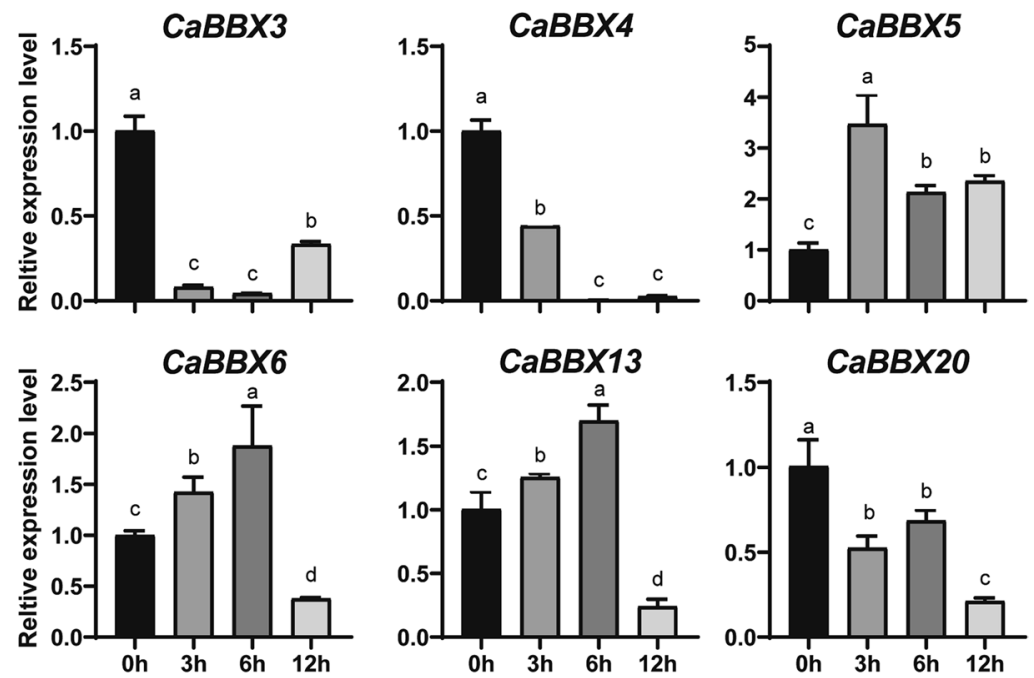

C
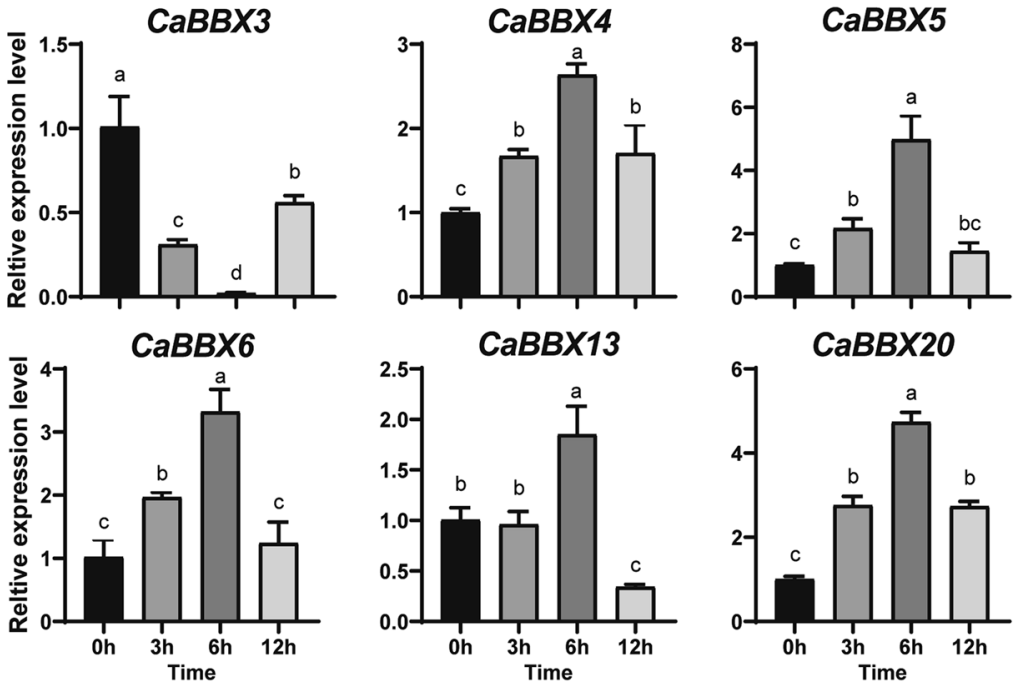

Fig. 8 The expression of CaBBXs under different hormone treatment. A. qRT-PCR transcript analysis of 6 selected CaBBX genes under ABA treatment. B. qRT-PCR transcript analysis of 6 selected CaBBX genes under MeJA treatment. C. qRT-PCR transcript analysis of 6 selected CaBBX genes under SA treatment. Three independent biological experiments were performed $(P<0.05)$ 


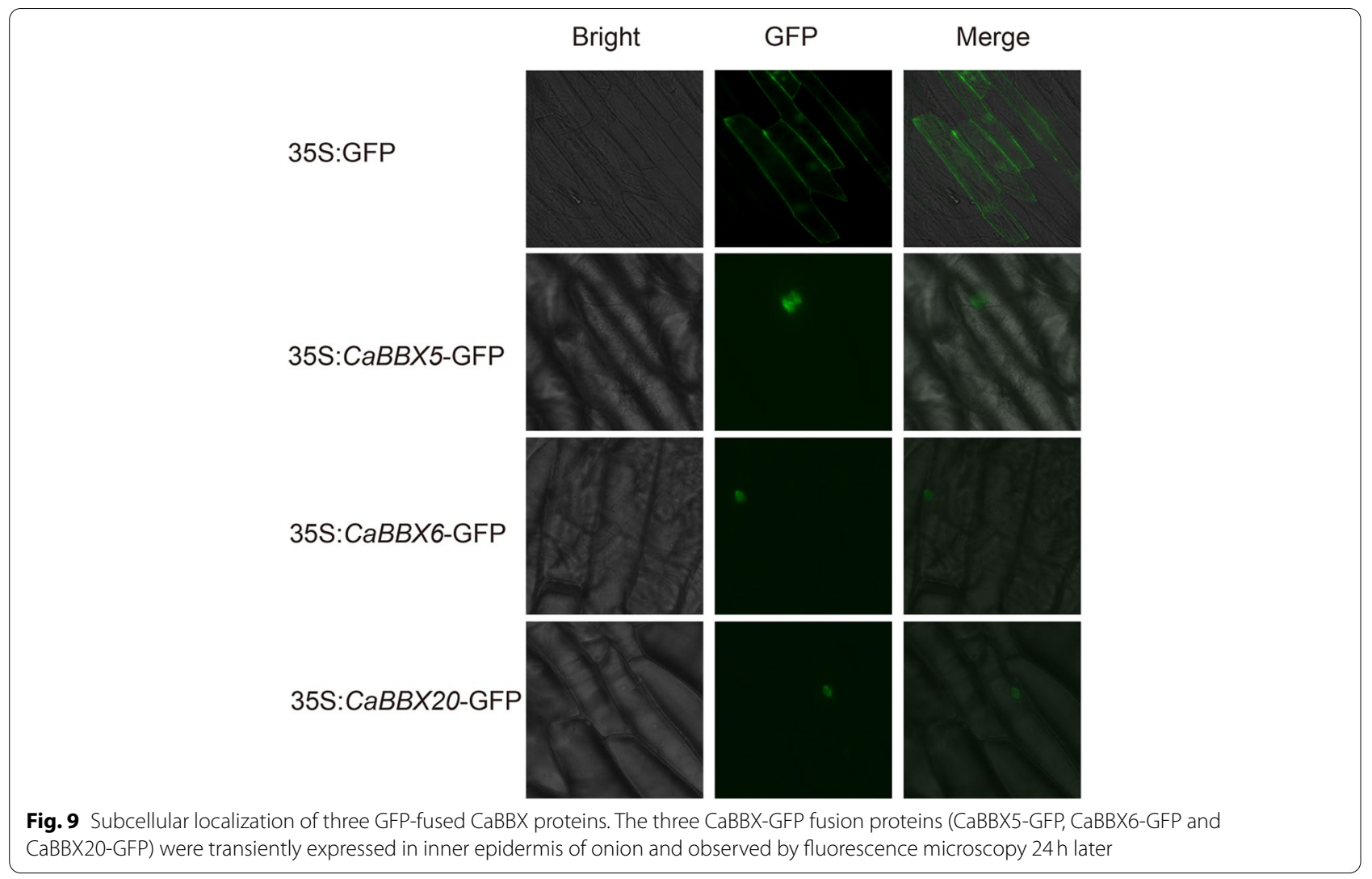

tomato [10], apple [13], pear [12], and so on [14, 42, 54]. The CaBBX was characterized the structure, phylogenetic relationship, chromosomal location, expression patterns and subcellular localization in our study. Based on the sequence similarity, the BBXs can be classified into five different subclasses [25]. The $B B X$ genes were investigated in 13 different high plants, and the total number among these plant species is relative stable with about 30 members (Fig. S4), expect for several species which experienced whole genome duplication. The triplication events occurred in Chinese cabbage genome since its divergence from Arabidopsis, resulting in the genome size of Chinese cabbage $(485 \mathrm{Mb})$ more than three times larger than that of Arabidopsis $(125 \mathrm{Mb})$ [55]. However, there were 75 BBX members in Chinese cabbage, only twice of that in Arabidopsis (32 members). Moreover, the number of GmBBXs (50 members) is more than three times of Medicago truncatula (15 members), soybean has undergone a specific tetraploidy [56]. The gene number is less than the genome duplication ploidy may result from alterative actions during the evolution. The number of $B B X$ genes in the three Solanaceae species (tomato, pepper, and potato) is relatively stable. However, the number of each subfamily among these plants differed a lot, this may indicate that the function of $\mathrm{BBX}$ belonging to different subclasses varied resulting from the differentiation of B-box proteins.

In addition, the diversity of are motif and intronexon structure important for the evolution of many gene families. In subclass I, except for CaBBX20, all the other six CaBBXs had six same motifs (motif 1,3 and 7). And CaBBX1-3 in a clustering clade within subclass II, contained all the same motif structure (Fig. 2A). It was identified that the CaBBXs in same phylogenetic clade shared the similar motif structures resulting in a conserved pattern in the evolution of $\mathrm{CaBBX}$ transcription factors.

Moreover, we found that the intron-exon structure of CaBBXs in same subclass also differed a little between the diverse clades, but are highly similar within the same phylogenetic clade. Thus, it is also indicated that the evolution of CaBBXs were relatively conserved with low occurrence of mutation events. However, the full genomic sequences of most $C a B B X$ genes were below $5 \mathrm{~kb}$, but not for that of $C a B B X 18$, 19 and 22 that were $6 \mathrm{~kb}, 7 \mathrm{~kb}$ and $10 \mathrm{~kb}$, respectively (Fig. 2B). Therefore, mutation events might still exist in highly conserved genes resulting from the evolutional diversity. 


\section{Gene duplication of BBX genes in pepper}

As is known, genetic novelty mainly caused by gene or genome duplication events, and gene family expansion was primarily resulting from gene duplication [57-59]. Novel BBX genes arise through divergence of duplicate genes after either single gene duplication, segmental duplication, or whole-genome duplication [57, 60]. The chromosomal location of $C a B B X$ genes indicated that the distribution of BBX genes in pepper genome is not even, this result may cause by genome duplication which occurred throughout plant evolution [61]. Three pairs of potential duplicate $B B X$ genes were found in pepper chromosomes, and a single $C a B B X$ gene, $C a B B X 7$, was located on ChrUM. Interestingly, all the three pairs of duplicate genes belonged to the same subgroup I, and $C a B B X 14$ duplicated with $C a B B X 15$ and $C a B B X 17$. While $C a B B X 17$ duplicated with $C a B B X 21$, which was clustered with $C a B B X 15$ located on chromosome 08 (Fig. 3). In addition, $26 C a B B X-S l B B X$ gene pairs were identified by collinearity analysis. Among them, 13 subgroup I gene pairs were found counted for the largest number of replication events. These results suggest that the expansion of CaBBX genes in pepper chromosome is affected by the network and duplication events of pepper.

The evolution of $\mathrm{CaBBX}$ transcription factors might indicate that their diverse function in tolerance to abiotic and biotic stress, responding to phytohormone, and even in plant growth and developmental processes, such as seedling photomorphogenesis, shade avoidance, photoperiodic regulation of flowering [25]. And the specific function of CaBBX transcription factors involved in plant development and stress tolerance were still looking forward to be elucidated.

\section{Tissue-specific and developmental expression patterns of CaBBX genes}

In several model plant, Arabidopsis, rice or tomato, the BBXs participated in seedling photomorphogenesis, such as flowering, hypocotyl growth, pigmentation and cotyledon unfolding $[8,27,33,62,63]$. Here, the transcript expression levels of $24 \mathrm{CaBBXs}$ were investigated in ten organs or tissues, as well as during leaf, flower, pulp, placenta and seed development (Fig. 5). Over-expression of CONSTANS-LIKE 5 can induce flowering under shortday condition in Arabidopsis [28]. AtBBX30 and AtBBX31 were negatively regulated by HY5, which directly binding to the G-box cis-element present in their promoters, negatively regulate photomorphogenesis in Arabidopsis [22]. While the homology of $A t B B X 30$ and $A t B B X 31$, $C a B B X 24$ was expressed at a high level in flower (Figs. 5 and 6). AtBBX4 is a key component involved in the phyB (Phytochrome B)-PIF3 (PHYTOCHROME INTERACTING FACTOR 3) regulatory module to promote photomorphogenesis [23]. CaBBX4 clustered with $A t B B X 4$, expressed highly in flower and pulp, while the flower and fruit development were related to photomorphogenesis. OsCO3, a $B B X$ gene in rice, can regulating flowering time by repressing the expression of FT-like genes under SD conditions [30]. Recent study suggested that AtBBX28 negatively regulates photomorphogenesis by repressing HY5 activity [21], the homologous $B B X$ in pepper is $C a B B X 23$, showed lower expression in flower in RNA-seq data transcript levels and qRT-PCR analysis (Figs. 5 and 6), with the similar function of AtBBX28. $C a B B X 7,12$ and 13 were expressed a relatively high level in the early stage of leaf development, may suggest they involved in cotyledon unfolding. In addition, $C a B B X 5$ and 6 were also expressed at a high level in both leaf and flower, suggesting they were involved in photomorphogenesis. CaBBX19 expressed only in seed, and had a gradually increasing expression pattern during seed development, indicating that its important roles in seed formation and growth. Besides, AtBBX24 and AtBBX25 were identified interacting with $\mathrm{HYH}$, an HY5 HOMOLOG, to regulate Arabidopsis seedling development [64], AtBBX24 and AtBBX25 were clustering with CaBBX20 by our phylogenetic analysis (Fig. S1). Recently, a tomato BBX transcription factor, SIBBX20 modulates fruit pigmentation by directly activating the rate-limited enzyme of carotenoid biosynthesis PSY1 [62]. CaBBX20 was classified into group I homologous with AtBBX24 and AtBBX25, and expressed a relatively high level in pepper pulp (Fig. 4), this may indicate that CaBBX20 evolved special function in fruit development.

\section{Stress and hormones induced expression of $B B X$ genes in pepper}

BBX transcription factors were also proved to be involved in response to stress and phytohormones [27, 35]. AtBBX18 was detected to be a negative regulator in heat tolerance in Arabidopsis [39]. In pepper, we found the expression profiles of $\mathrm{CaBBX} 4,5,6,13$ and 20 were significantly similar to be induced high expression by heat and salt stress. Moreover, $C a B B X 20$ homologous with $A t B B X 24$, was induced by salt stress (Fig. 7D), AtBBX24 also called STO, was identified to be a salt tolerance factor, which can enhance Arabidopsis root growth under salt stress treatment [35]. And it was found that SlBBX2O was up-regulated in M82 (cultivated tomato M82 is sensitive to stress) under drought stress in tomato [65]. Similarly, CaBBX4 was dramatically up-regulated (up to $10 \sim 60$ fold comparing with control) under drought stress, may indicated that they shared the similar function in responding to drought stress.

Phytohormones are important for plant growth, development and also involved in tolerance to biotic and 
abiotic stress. Recently, an apple B-box protein BBX37 was identified that regulates jasmonic acid mediated cold tolerance through the JAZ-BBX37-ICE1-CBF pathway [41], MdBBX10 significantly enhanced abiotic stresses tolerance by ABA signalling [66].BBX19 belonged to subgroup IV, interacts with $\mathrm{ABF} 3$ to affect drought tolerance negatively in chrysanthemum [67]. All these selected $B B X$ genes were found to be responded to $\mathrm{ABA}, \mathrm{MeJA}$ and SA. Except for $C a B B X 3$, other five $C a B B X$ genes were up-regulated expressed by ABA and SA application. This result may indicate that $C a B B X 4,5,6,13$ and 20 as positive factors response to $\mathrm{ABA}$ and SA signaling involved in pepper plant growth or biotic and abiotic stress tolerance. While, under MeJA condition, only CaBBX5 showed up-regulated expression at all three treated stages, others were down-regulated at different degrees, especially $C a B B X 3$ and 4 . This result may indicate that $C a B B X 3,4$, 6,13 and 20 as negative factors response to later stage of MeJA signaling.

\section{Conclusion}

In this study, we carried out a genome-wide analysis of $24 C a B B X$ genes, the phylogenetic analysis, domain, motif \& gene structure, gene chromosome location, were performed preliminarily. In addition, several $C a B B X$ genes were induced by abiotic stress and exogenous phytohormones, some expressed tissue-specific and variously at different developmental stage. And subcellular localization experiment was also investigated to further understand the potential function of $C a B B X$ genes, it was indicated that they act as nucleus-localized transcription factors. Overall, out data might be a foundation in the identification of $C a B B X$ genes, and a further understanding of their biological function in future studies.

\section{Supplementary Information}

The online version contains supplementary material available at https://doi. org/10.1186/s12864-021-08186-w.

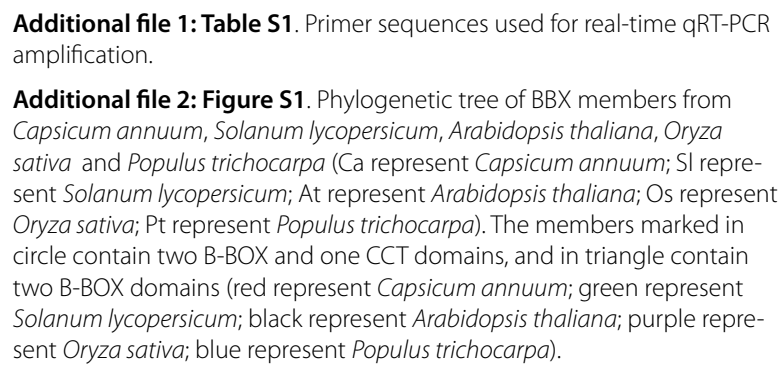

Additional file 2: Figure S1. Phylogenetic tree of BBX members from Capsicum annuum, Solanum lycopersicum, Arabidopsis thaliana, Oryza sativa and Populus trichocarpa (Ca represent Capsicum annuum; SI represent Solanum lycopersicum; At represent Arabidopsis thaliana; Os represent Oryza sativa; Pt represent Populus trichocarpa). The members marked in circle contain two B-BOX and one CCT domains, and in triangle contain two B-BOX domains (red represent Capsicum annuum; green represent Solanum lycopersicum; black represent Arabidopsis thaliana; purple represent Oryza sativa; blue represent Populus trichocarpa).

Additional file 3: Figure S2. Amino acid arrangement order of motif of BBX family.

Additional file 4: Figure S3. Expression profiles of CaBBX genes under different abiotic stresses. The first character $\mathrm{C}, \mathrm{F}, \mathrm{H}$ and $\mathrm{N}$ of the abbreviation on the bottom represent control, freezing, heat and $\mathrm{NaCl}$ treatment, respectively; the second character $L$ and $R$ represent leaf and root, respectively; the number $0,0.1$ and 0.2 represent 0 treatment; and the number $1,2,3,4,5$ and 6 represent $1 \mathrm{~h}, 1.5 \mathrm{~h}, 3 \mathrm{~h}, 6 \mathrm{~h}, 12 \mathrm{~h}$ and $24 \mathrm{~h}$ treatment.

Additional file 5: Figure S4. The classification of $B B X$ genes investigated in 13 higher plants (A-E representative five different subclasses in the BBXs Groups I V V, respectively).

\section{Acknowledgments}

We thank the Central Laboratory of Qingdao Agricultural University for the FluoView ${ }^{\mathrm{TM}}$ FV300 confocal microscope. We thank all of the teachers and students of the Key Laboratory of Horticultural Plant Genetic Improvement and Breeding of Qingdao for providing guidance and assistance.

\section{Authors' contributions}

$J M, D L, J X D$ and XWL conceived, designed and wrote the manuscript. JM analyzed data. JXD performed all experiments. All authors read and approved the final manuscript.

\section{Funding}

The work was supported by Agricultural Superior Seed Engineering Program of Shandong, China (2020LZGC005) and Shandong Agricultural Major Application Technology Innovation Project, China (6682218040). The funders had no role in the design of the study and collection, analysis, and interpretation of data and in writing the manuscript.

\section{Availability of data and materials}

All data generated or analyzed during this study are included in this article (and its supplementary information files) or are available from the corresponding author on reasonable request. Sequence data from this article can be found in Arabidopsis Information Resource (https://www.arabidopsis.org/) under the following accession numbers: AtBBX1 (AT5G15840), AtBBX2 (AT5G15850), AtBBX3 (AT3G02380), AtBBX4 (AT2G24790), AtBBX5 (AT5G24930), AtBBX6 (AT5G57660), AtBBX7 (AT3G07650), AtBBX8 (AT5G48250), AtBBX9 (AT4G15250), AtBBX10 (AT3G21880), AtBBX11 (AT2G47890), AtBBX12 (AT2G33500), AtBBX13 (AT1G28050), AtBBX14 (AT1G68520), AtBBX15 (AT1G25440), AtBBX16 (AT1G73870), AtBBX17 (AT1G49130), AtBBX18 (AT2G21320), AtBBX19 (AT4G38960), AtBBX20 (AT4G39070), AtBBX21 (AT1G75540), AtBBX22 (AT1G78600), AtBBX23 (AT4G10240), AtBBX24 (AT1G06040), AtBBX25 (AT2G31380), AtBBX26 (AT1G60250), AtBBX27 (AT1G68190), AtBBX28 (AT4G27310), AtBBX29 (AT5G54470), AtBBX30 (AT4G15248), AtBBX31 (AT3G21890), AtBBX32 (AT3G21150). The sequences of VvBBX1 (VIT_214s0083g00640.2), VvBBX2 (VIT_211s0052g01800.1), VvBBX3 (VIT_204s0008g07340.1), VvBBX4 (VIT_212s0057g01350.2), VvBBX5 (VIT_200s0194g00070.1), VvBBX6 (VIT_207s0104g01360.1), VvBBX7 (VIT_201s0146g00360.1), VvBBX8 (VIT_214s0068g01380.1), VvBBX9 (VIT_201s0011g04240.1), VvBBX10 (VIT_219s0014g05120.1), VvBBX11 (VIT_212s0059g02500.1), VvBBX12 (VIT_201s0011g03520.1), VvBBX13 (VIT_203s0038g00690.1), VvBBX14 (VIT_204s0023g03030.1), VvBBX15 (VIT_218s0001g13520.1), VvBBX16 (VIT_203s0038g00340.1), VvBBX17 (VIT_218s0089g01280.1), VvBBX18 (VIT_219s0014g00350.1), VvBBX19 (VIT_205s0102g00750.1), VvBBX20 (VIT_219s0014g03960.1), VvBBX21 (VIT_212s0134g00400.1), VvBBX22 (VIT_212s0059g02510.1), VvBBX23 (VIT_200s0203g00210.1), VvBBX24 (VIT_209s0054g00530.1) are available in Genome Annotation Batch Download of Grape Genome Annotation Project (http://genomes.cribi.unipd. it/grape/). The sequences of ZmBBX1 (zma100147736),

ZmBBX2(zma100281837), ZmBBX3 (zma100281289), ZmBBX4 (zma100281883), ZmBBX5 (zma100383826), ZmBBX6 (zma100281064), ZmBBX7 (zma100281114), ZmBBX8 (zma100193195), ZmBBX9 (zma100304421), ZmBBX10 (zma100282916), ZmBBX11 (zma100383648), ZmBBX12 (zma100283083), ZmBBX13 (zma100272654), ZmBBX14 (zma100283174), ZmBBX15 (zma100383388), ZmBBX16 (zma100273363), ZmBBX17 (zma100283103), ZmBBX18 (zma100284784), ZmBBX19 (zma100273793), ZmBBX20 (zma100274169), ZmBBX21 (zma100193074), ZmBBX22 (zma100284380), ZmBBX23 (zma100285359) are available in Genome Annotation Batch Download of maize Genome Annotation Project (http://www.maizesequence.org/index.html). The sequences of PtBBX1 
(POPTR_266027), PtBBX2 (POPTR_831202), PtBBX3 (POPTR_837482), PtBBX4 (POPTR_737847), PtBBX5 (POPTR_562026), PtBBX6 (POPTR_737468), PtBBX7 (POPTR_836808), PtBBX8 (POPTR_658893), PtBBX9 (POPTR_419707), PtBBX10 (POPTR_752788), PtBBX11 (POPTR_569981), PtBBX12 (POPTR_852783), PtBBX13 (POPTR_846848), PtBBX14 (POPTR_243653), PtBBX15 (POPTR_774835), PtBBX16 (POPTR_851021), PtBBX17 (POPTR_247140), PtBBX18 (POPTR_564570), PtBBX19 (POPTR_868551), PtBBX20 (POPTR_782168), PtBBX21 (POPTR_820080), PtBBX22 (POPTR_741179), PtBBX23 (POPTR_721866), PtBBX24 (POPTR_648693), PtBBX25 (POPTR_550941), PtBBX26 (POPTR_801530), PtBBX27 (POPTR_804174), PtBBX28 (POPTR_855496), PtBBX29 (POPTR_1094062), PtBBX30 (POPTR_1081997), PtBBX31 (POPTR_827145), PtBBX32 (POPTR_562313), PtBBX33 (POPTR_594447), PtBBX34 (POPTR_571834), PtBBX35 (POPTR_758524), PtBBX36 (POPTR_568988), PtBBX37 (POPTR_582065), PtBBX38 (POPTR_563458), PtBBX39 (POPTR_567790) are available in Genome Annotation Batch Download of Populus tomentosa Genome Annotation Project (http://www.genome.ad.jp/kegg/). The sequences of OsBBX1 (Os01g0202500), OsBBX2 (Os02g0176000), OsBBX3 (Os02g0178100), OsBBX4 (Os02g0606200), OsBBX5 (Os02g0610500), OsBBX6 (Os02g0646200), OsBBX7 (Os02g0724000), OsBBX8 (Os02g0731700), OsBBX9 (Os03g0351100), OsBBX10 (Os03g0711100), OsBBX11 (Os04g0493000), OsBBX12 (Os04g0497700), OsBBX13 (Os04g0540200), OsBBX14 (Os05g0204600), OsBBX15 (LOC_Os06g01340), OsBBX16 (Os06g0152200), OsBBX17 (Os06g0264200), OsBBX18 (Os06g0275000), OsBBX19 (Os06g0298200), OsBBX20 (Os06g0654900), OsBBX21 (Os06g0661200), OsBBX22 (Os06g0713000), OsBBX23 (Os07g0667300), OsBBX24 (Os08g0178800), OsBBX25 (Os08g0249000), OsBBX26 (Os08g0536300), OsBBX27 (Os09g0240200), OsBBX28 (Os09g0509700), OsBBX29 (Os09g0527900), OsBBX30 (Os12g0209200) are available in Genome Annotation Batch Download of Rice Genome Annotation Project (RGAP: http://rice.plant biol-ogy.msu.edu/). The sequences of MdBBX1 (MDP0000202669), MdBBX2 (MDP0000294359), MdBBX3 (MDP0000172036), MdBBX4 (MDP0000289278), MdBBX5 (MDP0000939920), MdBBX6 (MDP0000761905), MdBBX7 (MDP0000598183), MdBBX8 (MDP0000280947), MdBBX9 (MDP0000140460), MdBBX10 (MDP0000733075), MdBBX11 (MDP0000271388), MdBBX12 (MDP0000283949), MdBBX13 (MDP0000264228), MdBBX14 (MDP0000264845), MdBBX15 (MDP0000551876), MdBBX16 (MDP0000297093), MdBBX17 (MDP0000194889), MdBBX18 (MDP0000198531), MdBBX19 (MDP0000232355), MdBBX20 (MDP0000177126), MdBBX21 (MDP0000587860), MdBBX22 (MDP0000298804), MdBBX23 (MDP0000222881), MdBBX24 (MDP0000800387), MdBBX25 (MDP0000901915), MdBBX26 (MDP0000208320), MdBBX27 (MDP0000915501), MdBBX28 (MDP0000383112), MdBBX29 (MDP0000131980), MdBBX30 (MDP0000314259), MdBBX31 (MDP0000247810), MdBBX32 (MDP0000458656), MdBBX33 (MDP0000697407), MdBBX34 (MDP0000302297), MdBBX35 (MDP0000565292), MdBBX36 (MDP0000151848), MdBBX37 (MDP0000157816), MdBBX38 (MDP0000273201), MdBBX39 (MDP0000298575), MdBBX40 (MDP0000321380), MdBBX41 (MDP0000185616), MdBBX42 (MDP0000713113), MdBBX43 (MDP0000140484), MdBBX44 (MDP0000298635), MdBBX45 (MDP0000321180), MdBBX46 (MDP0000198072), MdBBX47 (MDP0000782323), MdBBX48 (MDP0000759984), MdBBX49 (MDP0000398010), MdBBX50 (MDP0000548690), MdBBX51 (MDP0000664576), MdBBX52 (MDP0000128581), MdBBX53 (MDP0000244238), MdBBX54 (MDP0000232445), MdBBX55 (MDP0000128008), MdBBX56 (MDP0000321735), MdBBX57 (MDP0000686172), MdBBX58 (MDP0000272743), MdBBX59 (MDP0000697109), MdBBX60 (MDP0000488955), MdBBX61 (MDP0000127949), MdBBX62 (MDP0000313949), MdBBX63 (MDP0000122414), MdBBX64 (MDP0000189746) are available in Genome Annotation Batch Download of Apple Genome Annotation Project (http://www.rosaceae.org/). The sequences of PbBBX1 (Pbr016562.1), PbBBX2 (Pbr023570.1), PbBBX3 (Pbr019957.1), PbBBX4 (Pbr036464.1), PbBBX5 (Pbr040252.1), PbBBX6 (Pbr022786.1), PbBBX7 (Pbr026954.1), PbBBX8 (Pbr038936.1), PbBBX9 (Pbr020281.1), PbBBX10 (Pbr013295.1), PbBBX11 (Pbr028831.1), PbBBX12 (Pbr022361.1), PbBBX13 (Pbr038976.1), PbBBX14 (Pbr042773.1), PbBBX15 (Pbr019591.1), PbBBX16 (Pbr015820.1), PbBBX17 (Pbr005884.1), PbBBX18 (Pbr020473.1), PbBBX19 (Pbr032616.1), PbBBX20 (Pbr034751.1), PbBBX21 (Pbr033352.1), PbBBX22 (Pbr011255.1), PbBBX23 (Pbr000255.1), PbBBX24
(Pbr022252.1), PbBBX25 (Pbr022252.1) are available in Genome Annotation Batch Download of Pear Genome Annotation Project (http://www.ebi.ac.uk/ Tools/pfa/iprscan/). In addition, the sequences of SIBBX1 (Solyc02g089520.1), SIBBX2 (Solyc02g089500.2), SIBBX3 (Solyc02g089540.2), SIBBX4 (Solyc08g006530.2), SIBBX5 (Solyc12g096500.1), SIBBX6 (Solyc07g006630.2), SIBBX7 (Solyc12g006240.1), SIBBX8 (Solyc05g020020.2), SIBBX9 (Solyc07g045180.2), SIBBX10 (Solyc05g046040.1), SIBBX11 (Solyc09g074560.2), SIBBX12 (Solyc05g024010.2), SIBBX13 (Solyc04g007210.2), SIBBX14 (Solyc03g119540.2), SIBBX15 (Solyc05g009310.2), SIBBX16 (Solyc12g005750.1), SIBBX17 (Solyc07g052620.1), SIBBX18 (Solyc02g084420.2), SIBBX19 (Solyc01g1 10370.2), SIBBX20 (Solyc12g089240.1), SIBBX21 (Solyc04g081020.2), SIBBX22 (Solyc07g062160.2), SIBBX23 (Solyc12g005420.1), SIBBX24 (Solyc06g073180.2), SIBBX25 (Solyc01g1 10180.2), SIBBX26 (Solyc10g006750.2), SIBBX27 (Solyc04g007470.2), SIBBX28 (Solyc12g005660.1), SIBBX29 (Solyc02g079430.2) are available in locus search of Sol Genomics Network (SGN:https://www.sgn. cornell.edu) database, and CaBBX1 (Capana02g003201), CaBBX2 (Capana02g003200), CaBBX3 (Capana02g003199), CaBBX4 (Capana01g004030), CaBBX5 (Capana12g000414), CaBBX6 (Capana07g000030), CaBBX7 (Capana00g004028), CaBBX8 (Capana07g001114), CaBBX9 (Capana00g004489), CaBBX10 (Capana03g003558), CaBBX11 (Capana00g001486), CaBBX12 (Capana11g002294), CaBBX13 (Capana03g000377), CaBBX14 (Capana02g002620), CaBBX15 (Capana08g002625), CaBBX16 (Capana12g000659), CaBBX17 (Capana04g000266), CaBBX18 (Capana07g002062), CaBBX19 (Capana09g000394), CaBBX20 (Capana06g000735), CaBBX21 (Capana08g002611), CaBBX22 (Capana05g001195), CaBBX23 (Capana07g001588), CaBBX24 (Capana00g004911) can be downloaded from the Pepper Genome Platform (PGP: http://peppergenome.snu.ac.kr/download. php).

\section{Declarations}

\section{Ethics approval and consent to participate}

Not applicable. This study was conducted in accordance with the People's Republic of China and international authorities' relevant guidelines and legislation, including the official website of the Committee on Publication Ethics (http://www.publicationethics.org/) and the European Association of Science Editors (EASE) and other institutions' publishing ethics standards. Pepper seeds ('Qingnong dried No.2') were obtained from the State Key Laboratory of Crop Genetics and Germplasm Enhancement in Qingdao Agricultural University. This cultivar was selected by researchers at Qingdao Agricultural University (Qingdao, China), the Qingdao Seed Station, and Dezhou Academy of Agricultural Sciences. Additionally, it was approved by the Shandong Variety Examination and Approval Committee in 2015(deposition number: 2015-057-1).

\section{Consent for publication}

Not applicable.

\section{Competing interests}

The authors declare that there are no competing interests.

Received: 17 May 2021 Accepted: 17 November 2021

Published online: 06 December 2021

\section{References}

1. Diao WP, Snyder JC, Wang SB, Liu JB, Pan BG, Guo GJ, et al. Genome-wide identification and expression analysis of WRKY gene family in Capsicum annuum L. Front Plant Sci. 2016;7:211.

2. Khanna R, Kronmiller B, Maszle DR, Coupland G, Holm M, Mizuno T, et al. The Arabidopsis B-box zinc finger family. Plant Cell. 2009;21(11):3416-20.

3. Meroni G, Diez-Roux G. TRIM/RBCC, a novel class of 'single protein RING finger'E3 ubiquitin ligases. BioEssays. 2005;27(11):1147-57.

4. Griffiths S, Dunford RP, Coupland G, Laurie DA. The evolution of CONSTANS-like gene families in barley, rice, and Arabidopsis. Plant Physiol. 2003:131(4):1855-67.

5. Robson F, Costa MM, Hepworth SR, Vizir I, Pineiro M, Reeves PH, et al. Functional importance of conserved domains in the flowering-time gene 
CONSTANS demonstrated by analysis of mutant alleles and transgenic plants. Plant J. 2001;28(6):619-31.

6. Yan $\mathrm{H}$, Marquardt K, Indorf M, Jutt D, Kircher S, Neuhaus G, et al. Nuclear localization and interaction with COP1 are required for STO/BBX24 function during photomorphogenesis. Plant Physiol. 2011;156(4):1772-82.

7. Gendron JM, Pruneda-Paz JL, Doherty CJ, Gross AM, Kang SE, Kay SA. Arabidopsis circadian clock protein, TOC1, is a DNA-binding transcription factor. Proc Natl Acad Sci U S A. 2012;109(8):3167-72.

8. Chang CS, Li YH, Chen LT, Chen WC, Hsieh WP, Shin J, et al. LZF1, a HY5regulated transcriptional factor, functions in Arabidopsis de-etiolation. Plant J. 2008;54(2):205-19.

9. Kumagai T, Ito S, Nakamichi N, Niwa Y, Murakami M, Yamashino T, et al. The common function of a novel subfamily of B-box zinc finger protein with reference to circadian-associated events in Arabidopsis thaliana. Biosci Biotechnol Biochem. 2008;72(6):1539-49.

10. Chu Z, Wang X, Li Y, Yu H, Li J, Lu Y, et al. Genomic organization, phylogenetic and expression analysis of the B-BOX gene family in tomato. Front Plant Sci. 2016;7:1552

11. Huang J, Zhao X, Weng X, Wang L, Xie W. The rice B-box zinc finger gene family: genomic identification, characterization, expression profiling and diurnal analysis. PLoS One. 2012;7(10):e48242.

12. Zou Z, Wang R, Wang R, Yang S, Yang Y. Genome-wide identification, phylogenetic analysis, and expression profiling of the BBX family genes in pear. J Hortic Sci Biotechnol. 2017;93(1):1-14.

13. Liu X, Li R, Dai Y, Chen X, Wang X. Genome-wide identification and expression analysis of the B-box gene family in the apple (Malus domestica Borkh.) genome. Mol Gen Genomics. 2018:293(2):303-15.

14. Shalmani A, Fan S, Jia P, Li G, Muhammad I, Li Y, et al. Genome identification of B-BOX gene family members in seven Rosaceae species and their expression analysis in response to flower induction in Malus domestica. Molecules. 2018;23(7):1763.

15. Onouchi H, Igeño MI, Périlleux C, Graves K, Coupland G. Mutagenesis of plants overexpressing CONSTANS demonstrates novel interactions among Arabidopsis flowering-time genes. Plant Cell. 2000;12(6):885-900.

16. Samach A, Onouchi H, Gold SE, Ditta GS, Schwarz-Sommer Z, Yanofsky MF, et al. Distinct roles of CONSTANS target genes in reproductive development of Arabidopsis. Science. 2000;288(5471):1613-6.

17. Ledger S, Strayer C, Ashton F, Kay SA, Putterill J. Analysis of the function of two circadian-regulated CONSTANS-LIKE genes. Plant J. 2001;26(1):15-22.

18. Xu D, Li J, Gangappa SN, Hettiarachchi C, Lin F, Andersson MX, et al. Convergence of light and ABA signaling on the ABI5 promoter. PLoS Genet. 2014:10(2):e1004197.

19. Xu D, Jiang Y, Li J, Lin F, Holm M, Deng XW. BBX21, an Arabidopsis B-box protein, directly activates HY5 and is targeted by COP1 for $26 S$ proteasome-mediated degradation. Proc Natl Acad Sci U S A. 2016;113(27):7655-60.

20. Xu D, Jiang Y, Li J, Holm M, Deng XW. The B-box domain protein BBX21 promotes Photomorphogenesis. Plant Physiol. 2018;176(3):2365-75.

21. Lin F, Jiang Y, Li J, Yan T, Fan L, Liang J, et al. B-BOX DOMAIN PROTEIN28 negatively regulates Photomorphogenesis by repressing the activity of transcription factor HY5 and undergoes COP1-mediated degradation. Plant Cell. 2018;30(9):2006-19.

22. Heng $Y$, Lin F, Jiang $Y$, Ding $M$, Yan $T$, Lan $H$, et al. B-box containing proteins $\mathrm{BBX} 30$ and $\mathrm{BBX} 31$, acting downstream of $\mathrm{HY} 5$, negatively regulate Photomorphogenesis in Arabidopsis. Plant Physiol. 2019;180(1):497-508.

23. Heng $Y$, Jiang $Y$, Zhao $X$, Zhou $H$, Wang $X$, Deng XW, et al. BBX4, a phyBinteracting and modulated regulator, directly interacts with PIF3 to fine tune red light-mediated photomorphogenesis. Proc Natl Acad Sci U S A. 2019;1 16(51):26049-56.

24. Zhao X, Heng Y, Wang X, Deng XW, Xu D. A positive feedback loop of BBX11-BBX21-HY5 promotes Photomorphogenic development in Arabidopsis. Plant Commun. 2020:1(5):100045.

25. Gangappa SN, Botto JF. The BBX family of plant transcription factors. Trends Plant Sci. 2014;19(7):460-70.

26. Xiao-Fei C, Zeng-Yu W. Overexpression of COL9, a CONSTANS-LIKE gene, delays flowering by reducing expression of $\mathrm{CO}$ and $\mathrm{FT}$ in Arabidopsis thaliana. Plant J. 2010;43(5):758-68.

27. Datta S, Hettiarachchi C, Johansson H, Holm M. SALT TOLERANCE HOMOLOG2, a B-box protein in Arabidopsis that activates transcription and positively regulates light-mediated development. Plant Cell. 2007:19(10):3242-55.
28. Miriam H, Yael H, Esther Y, Ido K, Rachel May G. Over-expression of CONSTANS-LIKE 5 can induce flowering in short-day grown Arabidopsis. Planta. 2009:230(3):481-91.

29. Park HY, Lee SY, Seok HY, Kim SH, Sung ZR, Moon YH. EMF1 interacts with EIP1, EIP6 or EIP9 involved in the regulation of flowering time in Arabidopsis. Plant Cell Physiol. 2011;52(8):1376-88.

30. Soon-Kap K, Choong-Hyo Y, Jeong Hwan L, Hee JY, Hyo-Young P, JeongKook K. OsCO3, a CONSTANS-LIKE gene, controls flowering by negatively regulating the expression of $\mathrm{FT}$-like genes under $\mathrm{SD}$ conditions in rice. Planta. 2008;228(2):355-65.

31. Yang-Seok L, Dong-Hoon J, Dong-Yeon L, Jakyung Y, Choong-Hwan R, Song $L$, et al. OsCOL4 is a constitutive flowering repressor upstream of Ehd1 and downstream of OsphyB. Plant J. 2010;63(1):18-30.

32. Yano M, Katayose Y, Ashikari M, Yamanouchi U, Monna L, Fuse T, et al. Hd1, a major photoperiod sensitivity quantitative trait locus in rice, is closely related to the Arabidopsis flowering time gene CONSTANS. Plant Cell. 2000;12(12):2473-84.

33. Yang $Y, M a C, X u Y$, Wei $Q$, Imtiaz $M$, Lan $H$, et al. A zinc finger protein regulates flowering time and abiotic stress tolerance in Chrysanthemum by modulating gibberellin biosynthesis. Plant Cell. 2014;26(5):2038-54.

34. Lippuner V, Cyert MS, Gasser CS. Two classes of plant CDNA clones differentially complement yeast calcineurin mutants and increase salt tolerance of wild-type yeast. J Biol Chem. 1996;271 (22):12859-66.

35. Nagaoka S, Takano T. Salt tolerance-related protein STO binds to a Myb transcription factor homologue and confers salt tolerance in Arabidopsis. J Exp Bot. 2003;54(391):2231-7.

36. Fujibe T, Saji H, Arakawa K, Yabe N, Takeuchi Y, Yamamoto KT. A methyl viologen-resistant mutant of Arabidopsis, which is allelic to ozone-sensitive $\mathrm{rcd} 1$, is tolerant to supplemental ultraviolet-B irradiation. Plant Physiol. 2004:134(1):275-85.

37. Belles-Boix E, Babiychuk E, Van Montagu M, Inze D, Kushnir S. CEO1, a new protein from Arabidopsis thaliana, protects yeast against oxidative damage. FEBS Lett. 2000;482(1-2):19-24

38. Jaspers $\mathrm{P}, \mathrm{Blomster} \mathrm{T}$, Brosche M, Salojarvi J, Ahlfors R, Vainonen JP, et al. Unequally redundant RCD1 and SRO1 mediate stress and developmental responses and interact with transcription factors. Plant J. 2009;60(2):268-79.

39. Wang Q, Tu X, Zhang J, Chen X, Rao L. Heat stress-induced BBX18 negatively regulates the thermotolerance in Arabidopsis. Mol Biol Rep. 2013;40(3):2679-88.

40. Takuhara Y, Kobayashi M, Suzuki S. Low-temperature-induced transcription factors in grapevine enhance cold tolerance in transgenic Arabidopsis plants. J Plant Physiol. 2011;168(9):967-75.

41. An JP, Wang XF, Zhang XW, You CX, Hao YJ. Apple B-box protein BBX37 regulates jasmonic acid mediated cold tolerance through the JAZBBX37-ICE1-CBF pathway and undergoes MIEL 1-mediated ubiquitination and degradation. New Phytol. 2020;229(5):2707-29.

42. Jingtao $H$, Yu R, Liping $G$. Identification and expression analysis of the B-box transcription factor family in pepper. Acta Horticulturae Sinica. 2021:48:1-15.

43. Qin C, Yu C, Shen Y, Fang X, Chen L, Min J, et al. Whole-genome sequencing of cultivated and wild peppers provides insights into Capsicum domestication and specialization. Proc Natl Acad Sci U S A. 2014;111(14):5135-40.

44. Liu F, Yu H, Deng Y, Zheng J, Liu M, Ou L, et al. PepperHub, an informatics hub for the chili pepper research community. Mol Plant. 2017:10(8):1129-32.

45. Gasteiger E, Gattiker A, Hoogland C, Ivanyi I, Appel RD, Bairoch A. ExPASy: the proteomics server for in-depth protein knowledge and analysis. Nucleic Acids Res. 2003;31(13):3784-8.

46. Chenna R, Sugawara H, Koike T, Lopez R, Gibson TJ, Higgins DG, et al. Multiple sequence alignment with the Clustal series of programs. Nucleic Acids Res. 2003:31(13):3497-500.

47. Tamura K, Peterson D, Peterson N, Stecher G, Nei M, Kumar S. MEGA5: molecular evolutionary genetics analysis using maximum likelihood, evolutionary distance, and maximum parsimony methods. Mol Biol Evol. 2011;28(10):2731-9.

48. Bailey TL, Boden M, Buske FA, Frith M, Grant CE, Clementi L, et al. MEME SUITE: tools for motif discovery and searching. Nucleic Acids Res. 2009:37(suppl 2):W202-8. 
49. Chen C, Chen H, Zhang Y, Thomas HR, Frank MH, He Y, et al. TBtools: An integrative toolkit developed for interactive analyses of big biological data. Mol Plant. 2020;13(8):1194-202.

50. Lee S, Hong JC, Jeon WB, Chung YS, Sung S, Choi D, et al. The salicylic acid-induced protection of non-climacteric unripe pepper fruit against Colletotrichum gloeosporioides is similar to the resistance of ripe fruit. Plant Cell Rep. 2009;28(10):1573-80.

51. Pfaffl MW. A new mathematical model for relative quantification in real-time RT-PCR. Nucleic Acids Res. 2001;29(9):e45.

52. Stern L. A visual approach to SPSS for windows : a guide to SPSS 17.0, 2nd edn. Boston: Allyn \& Bacon; 2010

53. Chou KC, Shen HB. Cell-PLoc: a package of web servers for predicting subcellular localization of proteins in various organisms. Nat Protoc. 2008;3(2):153-62.

54. Liu W, Tang R, Zhang Y, Liu X, Gao Y, Dai Z, et al. Genome-wide identification of B-box proteins and VvBBX44 involved in light-induced anthocyanin biosynthesis in grape (Vitis vinifera L.). Planta. 2021;253(5):114.

55. Wang $X$, Wang $H$, Wang J, Sun R, Wu J, Liu S, et al. The genome of the mesopolyploid crop species Brassica rapa. Nat Genet. 2011:43(10):1035-9.

56. Wang J, Sun P, Li Y, Liu Y, Yu J, Ma X, et al. Hierarchically aligning 10 legume genomes establishes a family-level genomics platform. Plant Physiol. 2017;174(1):284-300.

57. Taylor JS, Raes J. Duplication and divergence: the evolution of new genes and old ideas. Annu Rev Genet. 2004;38:615-43.

58. Moore RC, Purugganan MD. The evolutionary dynamics of plant duplicate genes. Curr Opin Plant Biol. 2005;8(2):122-8.

59. Semon M, Wolfe KH. Consequences of genome duplication. Curr Opin Genet Dev. 2007;17(6):505-12

60. Kellogg EA. What happens to genes in duplicated genomes. 2003:100(8):4369-71.

61. Cannon SB, Mitra A, Baumgarten A, Young ND, May G. The roles of segmental and tandem gene duplication in the evolution of large gene families in Arabidopsis thaliana. BMC Plant Biol. 2004;4:10

62. Xiong C, Luo D, Lin A, Zhang C, Shan L, He P, et al. A tomato B-box protein SIBBX20 modulates carotenoid biosynthesis by directly activating PHYTOENE SYNTHASE 1, and is targeted for 265 proteasome-mediated degradation. New Phytol. 2019;221(1):279-94.

63. Datta S, Johansson H, Hettiarachchi C, Irigoyen ML, Desai M, Rubio V, et al. LZF1/SALT TOLERANCE HOMOLOG3, an Arabidopsis B-box protein involved in light-dependent development and gene expression, undergoes COP1-mediated ubiquitination. Plant Cell. 2008:20(9):2324-38.

64. Gangappa SN, Holm M, Botto JF. Molecular interactions of BBX24 and BBX25 with HYH, HY5 HOMOLOG, to modulate Arabidopsis seedling development. Plant Signal Behav. 2013;8(8):e25208.

65. Gong P, Zhang J, Li H, Yang C, Zhang C, Zhang X, et al. Transcriptional profiles of drought-responsive genes in modulating transcription signal transduction, and biochemical pathways in tomato. J Exp Bot. 2010;61(13):3563-75.

66. Liu X, Li R, Dai Y, Yuan L, Sun Q, Zhang S, et al. A B-box zinc finger protein, MdBBX10, enhanced salt and drought stresses tolerance in Arabidopsis. Plant Mol Biol. 2019;99(4-5):437-47.

67. Xu Y, Zhao X, Aiwaili P, Mu X, Zhao M, Zhao J, et al. A zinc finger protein BBX19 interacts with ABF3 to affect drought tolerance negatively in chrysanthemum. Plant J. 2020;103(5):1783-95.

\section{Publisher's Note}

Springer Nature remains neutral with regard to jurisdictional claims in published maps and institutional affiliations. 